\title{
Two-dimensional simulation by regularization of free surface viscoplastic flows with Drucker-Prager yield stress and application to granular collapse
}

\author{
Christelle Lusso ${ }^{1}$, Alexandre Ern ${ }^{1}$, François Bouchut ${ }^{2 *}$, Anne Mangeney ${ }^{3,4}$, \\ Maxime Farin ${ }^{3}$ and Olivier Roche ${ }^{5}$
}

\author{
${ }^{1}$ Université Paris-Est, CERMICS (ENPC), F-77455, Marne-la-Vallée cedex 2, France \\ ${ }^{2}$ Université Paris-Est, Laboratoire d'Analyse et de Mathématiques Appliquées (UMR 8050), \\ CNRS, UPEM, UPEC, F-77454, Marne-la-Vallée, France \\ ${ }^{3}$ Université Paris Diderot, Sorbonne Paris Cité, Institut de Physique du Globe de Paris, \\ Seismology group, 1 rue Jussieu, 75005 Paris, France, \\ ${ }^{4}$ ANGE team, INRIA, CETMEF, Lab. J.-Louis Lions, Paris, France, \\ ${ }^{5}$ Laboratoire Magma et Volcans, Université Blaise Pascal-CNRS-IRD, OPGC, Campus des \\ Cézeaux, 6 avenue Blaise Pascal, 63178 Aubière, France
}

\begin{abstract}
This work is devoted to numerical modeling and simulation of granular flows relevant to geophysical flows such as avalanches and debris flows. We consider an incompressible viscoplastic fluid, described by a rheology with pressure-dependent yield stress, in a $2 \mathrm{D}$ setting with a free surface. We implement a regularization method to deal with the singularity of the rheological law, using a mixed finite element approximation of the momentum and incompressibility equations, and an arbitrary Lagrangian Eulerian (ALE) formulation for the displacement of the domain. The free surface is evolved by taking care of its deposition onto the bottom and of preventing it from folding over itself. Several tests are performed to assess the efficiency of our method. The first test is dedicated to verify its accuracy and cost on a one-dimensional simple shear plug flow. On this configuration we setup rules for the choice of the numerical parameters. The second test aims to compare the results of our numerical method to those predicted by an augmented Lagrangian formulation in the case of the collapse and spreading of a granular column over a horizontal rigid bed. Finally we show the reliability of our method by comparing numerical predictions to data from experiments of granular collapse of both trapezoidal and rectangular columns over horizontal rigid or erodible granular bed made of the same material. We compare the evolution of the free surface, the velocity profiles, and the static-flowing interface. The results show the ability of our method to deal numerically with the front behavior of granular collapses over an erodible bed.
\end{abstract}

Keywords. Viscoplastic flows, Drucker-Prager pressure-dependent yield stress, free surface, regularization, ALE method, granular collapse

\section{Introduction}

Gravity-driven flows of granular material [2] are encountered in many industrial processes. Such flows are also particularly important in geophysics since they often represent natural hazards [17]. The understanding of these geophysical flows is a key issue for the description

\footnotetext{
*Corresponding author. Email: francois.bouchut@u-pem.fr
} 
and prediction of catastrophic events such as rock, debris or snow avalanches, landslides and pyroclastic flows. In order to predict landslide dynamics and deposition [46], the rheological behavior of natural granular flows must be known and this has motivated many studies and is still an open question, e.g., [59, 46, 26, 54, 17]. Even at laboratory scale, the constitutive equations for granular flows are still debated [16, 15, 27, 30, 41, 52, 14, 35]. One major complication is that flows of dense granular materials present zones at rest and flowing zones within the mass, which implies that granular materials can behave like a solid or flow like a liquid. The liquid state, which has been the subject of a wide range of research $[1,30]$, still lacks a unified view and the physical interface between the no-flow (static) and flow zones (flowing) is a central issue in granular material research. In most granular flows, the static-flowing transition represents a key to understanding natural flow dynamics. From a geophysical point of view, the transition also plays a crucial role in erosion-deposition processes.

It has been recognized that, in many situations, the behavior of granular materials is similar to classical viscoplastic materials [30, 15, 27]. A variety of constitutive equations have been proposed in the literature for modeling the rheological behavior of these viscoplastic materials. The viscoplastic laws are characterized by a yield criterion, which means that the material flows in regions where the stress is larger than a critical yield stress and is static in regions where the stress remains below this yield limit. In our framework, the threshold value is deduced from a pressure-dependent criterion such as the Mohr-Coulomb criterion. It is the Drucker-Prager formula [20] that states that the yield stress is proportional to the pressure. In [41], it has been proposed that this type of viscoplastic model is well suited for the description of dense granular material flows.

Viscoplastic flows are described mathematically by variational inequalities $[28,22,33,62]$. Two main families of numerical methods are available to deal with such inequalities. The first method uses a dual approach based on an augmented Lagrangian [32, 58, 9, 36, 35]. It is well suited to the main mathematical difficulty associated with yield stress models, that is, the non-differentiability of the stress-strain rate relation at the yield point. The second method consists in approximating the equation modeling the yield stress behavior by a smooth relationship. It is called the regularization method and is based on the introduction of an additional parameter $[5,56,44,29,18,42,19,6,11]$. The augmented Lagrangian method is supposed to better resolve the static-flowing interface [63] (although this resolution is anyway affected by discretization errors). In the present work we have chosen to use the regularization method because its implementation is straightforward so that this method is often used in practice [29]. Our aim is not to compare one method against the other, but to show that the regularization method can yield reliable results at attractive computational costs when simulating granular collapses in various configurations. Important issues addressed in our study are the choice of the regularization parameter and the criterion for determining the static-flowing interface. These are described in Subsection 5.1.

In a natural context, granular flows usually travel on granular deposits built up by earlier events. The layer of particles flowing over the initially static layer may entrain material of the static bed, which may or may not be made of the same grains (erosion process). The entrainment of underlying material may have a significant impact on granular flows. In particular, it is expected to modify the dynamics of the flowing mass for slope angles exceeding a critical value about equal to half the repose angle, and thereby significantly increasing the runout distance (i.e., the maximum distance traveled by the flow) [51, 52, 57,61 . Natural granular flows are mainly characterized by their final deposit, shape and runout distance. However due to the difficulty of conducting field measurements of material entrainment in nature, the physical understanding of flow dynamics and erosion processes remains incomplete. The theoretical description of such entrainment phenomena is likewise an open problem. For this reason, laboratory experiments of granular flows are a useful way to obtain new insights into the erosion/deposition processes. Experiments involving the collapse of granular columns over rigid beds first made it possible to establish scaling laws to investigate flow dynamics and deposits, as well as to test constitutive relations, see, e.g., 
$[3,43,45,49,35]$. Other experiments on granular collapse over erodible beds have been conducted to investigate and quantify erosion processes [14, 25, 51]. These studies have revealed that, for slope angles above a critical threshold about equal to half the repose angle, the presence of even a thin layer of erodible bed increases the flow duration and the runout distance compared to that of collapse over a rigid bed, while also changing the flow regimes $[25,37,51]$. While many numerical studies have focused on the reproduction of experimental scaling laws for granular collapse over rigid beds, few attempts have been made to incorporate the entrainment processes so as to simulate the propagation of granular flows over erodible beds $[52,7,13,14,38,39]$. However, such simulations are of primary importance, especially for the prediction of the runout length of geophysical flows.

We propose here to simulate the collapse of granular columns over a horizontal, rigid or erodible bed. We consider an incompressible viscoplastic flow with a rheology described by a pressure-dependent Drucker-Prager yield stress, together with friction and free surface boundary conditions in a two-dimensional setting. We do not consider surface tension effects at the free surface (in some cases, we add a small tension, but only as a numerical tool). The regularization method is implemented with Taylor-Hood finite elements for space discretization and an implicit Euler scheme with linearization for time discretization, similarly to the implementation in [11]. Additionally, an Arbitrary Lagrangian Eulerian (ALE) method is used as in [35] to deal with the displacement of the domain, in particular to track the free surface. Two numerical tools are used in order to deal with a proper displacement of the free surface. The first is a specific treatment of the deposition of the front onto the rigid bed, illustrated on Figure 2. The second, in the case of erodible bed, is the use of a local surface tension at the leading tip of the falling mass to handle the folding of the free surface onto itself that is observed experimentally, and replace it by a straight free surface, see Figure 4 (right). Without these treatments, the ALE method would not be able to deal with the granular collapse configuration. Our study is the first to consider the regularization method together with the ALE method to deal with a free surface and an erodible bed. Note that $[42,19]$ use the finite volume method on a fixed mesh, with, for tracking the free surface, either the immersed boundary method in [42] or the marker-and-cell method in [19]. In [10] it is shown that the use of the pure Lagrange method to move the mesh leads to instabilities. In addition, our study is, to our knowledge, the first numerical investigation of the collapse of a trapezoidal mass over an erodible bed.

The paper is organized as follows. In Section 2 we present the governing equations of the two-dimensional, incompressible, viscoplastic model with Drucker-Prager yield stress. We also introduce the geometry of the domain and the regularization method. The numerical implementation based on the Arbitrary Lagrangian Eulerian method is described in Section 3. There we describe the numerical algorithms devised for the deposition of the free surface onto the rigid bottom or for managing the folding of the free surface by a local numerical surface tension in the case of erodible bed. We devote Section 4 to a quantitative comparison with a one-dimensional simple shear plug flow over an inclined planar bed, for which we evaluate the convergence error by extending the one-dimensional solution to a two-dimensional setting with periodic boundary conditions. In Section 5 we consider the collapse of rectangular and trapezoidal granular columns. We describe in detail our choice of the regularization parameter and of the time step, and the criterion for determining the static-flowing interface. We compare the predicted velocity, interface and thickness profiles with those predicted by an augmented Lagrangian method [35] and with results from laboratory experiments [25]. The regularization method is shown to provide results in good overall agreement with experimental measurements and with the augmented Lagrangian method, while requiring significantly less computational time than the latter. 


\section{Mathematical formulation}

\subsection{Viscoplastic model with Drucker-Prager yield stress}

We consider a two-dimensional viscoplastic flow over an inclined slope of angle $\theta$. The material fills a time-dependent domain denoted by $\Omega_{t}$, with $t \in[0, T]$, and $T>0$ a given time. The position vector in $\Omega_{t}$ is denoted by $\vec{X}$. We consider two different configurations (see Figure 1): (a) a periodic flow over an inclined bed and (b) the collapse of a granular mass over a horizontal, rigid or erodible bed. In the experiments with erodible bed there is initially a thin layer of granular material, which is entrained by the expanding granular mass. Such a thin layer is not present in the rigid bed experiments. These two configurations (a), (b) lead to different initial and boundary conditions. We first write the mass and momentum conservation equations together with the constitutive equations, and then mention the particular geometry and boundary conditions for each configuration.

The material is assumed to be incompressible and non-Newtonian,

$$
\begin{aligned}
\rho\left(\partial_{t} \boldsymbol{u}+(\boldsymbol{u} \cdot \nabla) \boldsymbol{u}\right)-\operatorname{div} \boldsymbol{\sigma} & =\rho \boldsymbol{f} & & \text { for } t \in(0, T), \vec{X} \in \Omega_{t}, \\
\operatorname{div} \boldsymbol{u} & =0 & & \text { for } t \in(0, T), \vec{X} \in \Omega_{t},
\end{aligned}
$$

where $\boldsymbol{u}$ is the material velocity, $\rho>0$ the mass density, and $\boldsymbol{\sigma}$ the total stress tensor. The force $\boldsymbol{f}$ is the gravity force acting on the material. It is given by $\boldsymbol{f}=(g \sin \theta,-g \cos \theta)$ in the coordinates $(X, Z)$, with $g$ the gravity constant and $\theta>0$ the slope angle of the possibly inclined bed (see Figure 1).
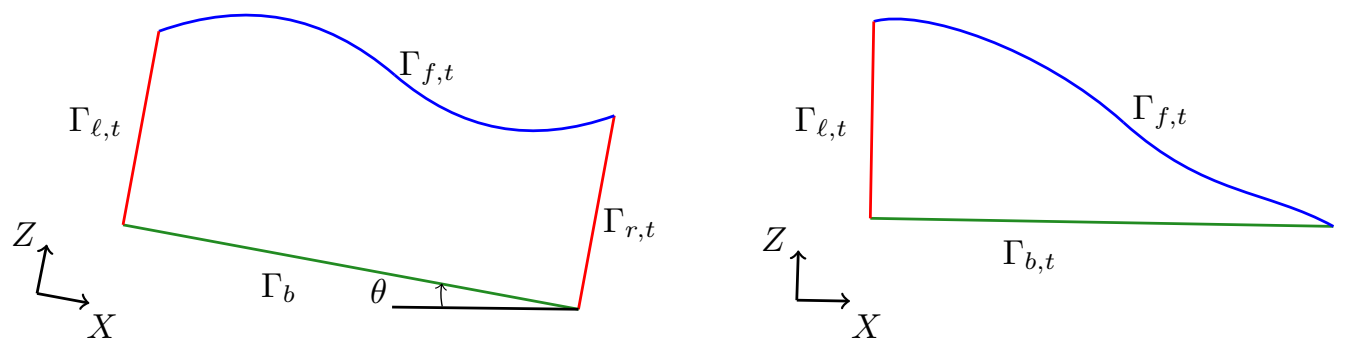

Figure 1: Domain $\Omega_{t}$ occupied by the material at time $t$. Left: periodic flow over an inclined bed (configuration (a)); right: granular collapse over a horizontal rigid or erodible bed (configuration (b)).

The rheology is defined by the viscoplastic law

$$
\boldsymbol{\sigma}=\boldsymbol{\sigma}^{\prime}-p \mathrm{Id}, \quad \text { with } \boldsymbol{\sigma}^{\prime}=2 \eta D \boldsymbol{u}+\kappa \frac{D \boldsymbol{u}}{\|D \boldsymbol{u}\|},
$$

where $\boldsymbol{\sigma}^{\prime}$ denotes the deviatoric part of $\boldsymbol{\sigma}, p$ is the pressure, $D \boldsymbol{u}=\frac{1}{2}\left(\nabla \boldsymbol{u}+\nabla \boldsymbol{u}^{t}\right)$ is the strain rate tensor, $\eta \geq 0$ is the dynamic viscosity, and $\kappa \geq 0$ is the yield stress. Here, the norm is the unmodified Frobenius norm $\|D \boldsymbol{u}\|^{2}=\sum_{i j}(D \boldsymbol{u})_{i j}^{2}$. We do not put a factor $1 / 2$ in the definition of this norm, as many authors do; this leads to the factor $\sqrt{2}$ in the formulas below. The viscoplastic constitutive relation can be written more rigorously as

$$
\operatorname{trace}\left(\boldsymbol{\sigma}^{\prime}\right)=0, \quad \begin{cases}\boldsymbol{\sigma}^{\prime}=2 \eta D \boldsymbol{u}+\kappa \frac{D \boldsymbol{u}}{\|D \boldsymbol{u}\|} & \text { if } D \boldsymbol{u} \neq 0 \\ \left\|\boldsymbol{\sigma}^{\prime}\right\| \leq \kappa & \text { if } D \boldsymbol{u}=0\end{cases}
$$

In general, in (2.2) or (2.3), $\eta$ and $\kappa$ can be rate- and pressure-dependent, i.e., they can depend on $\|D \boldsymbol{u}\|$ and $p$. Assuming that the free surface separates the material from air at 
the reference zero pressure and taking into account a possibly negative pressure inside the mass, the Drucker-Prager yield stress [20] can be written

$$
\kappa(p)=\sqrt{2} \mu_{s}[p]_{+},
$$

where $\mu_{s} \geq 0$ is the static internal friction coefficient, a constant depending only on the material, and $[p]_{+}=\max (0, p)$. The viscosity $\eta$ is taken constant in this work, although the value $\eta=\left(\mu(I)-\mu_{s}\right)[p]_{+} / \sqrt{2}\|D \boldsymbol{u}\|$ with $I \sim\|D \boldsymbol{u}\| / \sqrt{p}$ could be taken also, for the case of the $\mu(I)$ rheology of [41], as considered in [11, 35]. It has been shown in [35] for granular collapses over rigid beds that taking a constant viscosity does not change much the results as soon as the value of the viscosity is well chosen, i.e. deduced from the average of the values of the viscosity derived from the $\mu(I)$ rheology. Numerical experiments indicate that choosing a constant viscosity generally improves the stability and the efficiency of the simulation. When using a $\mu(I)$ rheology, numerical difficulties can arise, e.g., related to the movement of the mesh or to the non-convergence of some iterative processes. While the origin of these difficulties deserves further study (see [11] in the steady case), the choice of constant viscosity appears therefore to be more adapted to our objective of evaluating the coupling of the mesh displacement with the regularization of the Drucker-Prager rheology.

Let us briefly recall some mathematical results. The Bingham model $\kappa=c s t$ is always well-posed [33], even without viscosity [6]. The model with Drucker-Prager yield stress and without viscosity is linearly ill-posed [60] (which means that the system linearized around a given solution is ill-posed). The model with $\mu(I)$ from [41] is linearly ill-posed for small or large $I$ [4]. The model with positive constant viscosity $\eta$ considered here is linearly well-posed if $2 \eta\|D \boldsymbol{u}\| \geq \sqrt{2} \mu_{s} p$, as can be seen with a simple adaptation of the computations of [4], see [53]. A model with pressure-dependent yield stress and regularization is however proved to be well-posed in the steady case with small friction in [23], and the case with shear in a $2 \mathrm{D}$ channel without regularization is analyzed in [55]. The physical meaning of these well or ill-posedness results remains however unclear.

The equations (2.1) are supplemented with boundary conditions which depend on the considered configuration.

(a) Periodic flow over an inclined bed: This configuration is only used for the purpose of numerical verification. The domain $\Omega_{t}$ is composed of a flat, time-independent bottom $\Gamma_{b}$, a free surface $\Gamma_{f, t}$, and lateral boundaries $\Gamma_{\ell, t}$ and $\Gamma_{r, t}$. We assume a no-slip condition at the bottom

$$
\boldsymbol{u}(t, \vec{X})=\mathbf{0} \quad \text { for } t \in(0, T), \vec{X} \in \Gamma_{b},
$$

and on the lateral boundaries we impose periodicity conditions as

$$
\begin{aligned}
\boldsymbol{u}(t, \vec{X})=\boldsymbol{u}(t, \mathcal{T}(\vec{X})) & \text { for } t \in(0, T), \vec{X} \in \Gamma_{\ell, t}, \\
(\boldsymbol{\sigma}(t, \vec{X})-\boldsymbol{\sigma}(t, \mathcal{T}(\vec{X}))) \boldsymbol{N}=\mathbf{0} & \text { for } t \in(0, T), \vec{X} \in \Gamma_{\ell, t}
\end{aligned}
$$

where $\boldsymbol{N}$ stands for the outward unit normal on the boundary of the domain $\Omega_{t}$, and $\mathcal{T}$ is the translation in space such that $\mathcal{T}\left(\Gamma_{\ell, t}\right)=\Gamma_{r, t}$.

(b) Granular collapse over a horizontal rigid or erodible bed: The boundary of the domain $\Omega_{t}$ is composed of a flat bottom $\Gamma_{b, t}$, a free surface $\Gamma_{f, t}$, and a left wall $\Gamma_{\ell, t}$. In the experiments, the bed is covered by particles glued on its surface that are the same as those released from the column. In the case of an erodible bed, this rough bottom is itself covered by a layer of particles that can be entrained by the flow. The erodible bed is part of the simulation domain, so that in all cases, the bottom boundary of the domain is the rigid rough bed, see Figure 6 . The boundary condition for granular flows with rough bed or plastic wall is expected to be better described by a Coulomb friction law than with a no-slip condition. This is the case both in continuum models [36, 40] and in discrete methods [31]. We thus consider the following boundary conditions. 
At the bottom and at the left wall, we consider a non-penetration condition

$$
\boldsymbol{u}(t, \vec{X}) \cdot \boldsymbol{N}=0 \quad \text { for } t \in(0, T), \vec{X} \in \Gamma_{b, t} \cup \Gamma_{\ell, t},
$$

together with a Coulomb friction condition

$$
\begin{array}{ll}
\boldsymbol{\sigma}_{T}^{\prime}=-\mu_{b / \ell} \frac{\boldsymbol{u}_{T}}{\left|\boldsymbol{u}_{T}\right|}\left[p-\sigma_{N}^{\prime}\right]_{+} & \text {if } \boldsymbol{u}_{T} \neq 0, \text { for } t \in(0, T), \vec{X} \in \Gamma_{b, t} \cup \Gamma_{\ell, t}, \\
\left|\boldsymbol{\sigma}_{T}^{\prime}\right| \leq \mu_{b / \ell}\left[p-\sigma_{N}^{\prime}\right]_{+} & \text {if } \boldsymbol{u}_{T}=0, \text { for } t \in(0, T), \vec{X} \in \Gamma_{b, t} \cup \Gamma_{\ell, t} .
\end{array}
$$

with a friction coefficient $\mu_{b / \ell}=\mu_{b}$ on the bottom and $\mu_{b / \ell}=\mu_{\ell}$ on the left wall, and where the normal and tangential decomposition of $\boldsymbol{u}$ and $\boldsymbol{\sigma}^{\prime} \boldsymbol{N}$ are

$$
\begin{array}{rlll}
\boldsymbol{u} & =u_{N} \boldsymbol{N}+\boldsymbol{u}_{T} & \text { with } & u_{N}=\boldsymbol{u} \cdot \boldsymbol{N} \\
\boldsymbol{\sigma}^{\prime} \boldsymbol{N} & =\sigma_{N}^{\prime} \boldsymbol{N}+\boldsymbol{\sigma}_{T}^{\prime} \quad \text { with } & \sigma_{N}^{\prime} & =\left(\boldsymbol{\sigma}^{\prime} \boldsymbol{N}\right) \cdot \boldsymbol{N}
\end{array}
$$

Note that (2.7) implies that $u_{N}=0$ at the bottom and left boundaries.

Moreover, in configurations (a) and (b), we assume a no-stress condition at the free surface

$$
\boldsymbol{\sigma}(t, \vec{X}) \boldsymbol{N}=\gamma \boldsymbol{N} \quad \text { for } t \in(0, T), \vec{X} \in \Gamma_{f, t}
$$

where $\gamma$ is a real number that will be taken non-zero for numerical purpose only in Subsection 3.4. This artificial surface tension is just a numerical device to deal with the folding of the granular mass near the front, and is taken small enough not to disturb the flow as explained in Subsection 3.4. We also have the following kinematic condition at the free surface, that governs the evolution of the domain $\Omega_{t}$ :

$$
N_{t}+\boldsymbol{N} \cdot \boldsymbol{u}(t, \vec{X})=0 \quad \text { for } t \in(0, T), \vec{X} \in \Gamma_{f, t},
$$

where $\left(N_{t}, \boldsymbol{N}\right)$ is the time-space normal at the free surface.

Finally, the initial condition is $\boldsymbol{u}(0, \vec{X})=\boldsymbol{u}_{0}(\vec{X})$ for $\vec{X} \in \Omega_{0}$ with given $\boldsymbol{u}_{0}$ and $\Omega_{0}$.

\subsection{Regularization and variational formulation}

The main difficulty in the viscoplastic rheology (2.2) is the presence of yield stress, which implies the non-differentiability of the stress-strain rate relation at the yield point, and the indeterminate nature of the stress field below the yield point. The numerical treatment of the multi-valued term $\kappa D \boldsymbol{u} /\|D \boldsymbol{u}\|$ in (2.2) is usually performed by two methods. The first approach is to use duality methods, the main one being the augmented Lagrangian formulation. In the case of a Bingham fluid $(\kappa=c s t)$, this is well explained in [58], and simulations with free surface are performed in [35] for a Drucker-Prager yield stress. The other approach, that we adopt here, is the regularization method. Although it is slightly less accurate than the augmented Lagrangian method, it has the advantage to be easier to formulate, and to be computationally more effective $[56,44,29,18,11]$. The regularization method consists in approximating the non-smooth constitutive law with yield stress by a smoother one without yield stress. It is achieved through the introduction of a parameter $\epsilon>0$, and the regularized constitutive relation is written in the form

$$
\boldsymbol{\sigma}_{\epsilon}^{\prime}=2 \eta D \boldsymbol{u}+\kappa \frac{D \boldsymbol{u}}{\sqrt{\|D \boldsymbol{u}\|^{2}+\epsilon^{2}}} .
$$

In this formulation, the right-hand side is always well-defined, even if $D \boldsymbol{u}$ vanishes. However, the expression (2.13) can differ from (2.2) when $\|D \boldsymbol{u}\|$ is of the order of $\epsilon$, or less. Thus the accuracy in determining the plug zone where $D \boldsymbol{u}=0$ is strongly related to the smallness of $\epsilon$ [63]. Generally speaking, $\epsilon$ has to be taken sufficiently small so that the regularization error does not dominate over the space discretization error. Then the plug zone can be defined as 
the locations where $D \boldsymbol{u}$ is smaller than the typical approximation error. For the Bingham model $\kappa=c s t$, the convergence as $\epsilon \rightarrow 0$ is proved in [33, p. 370] in the case with viscosity and $H^{1}$ regularity, at rate $\epsilon$, and in the case without viscosity and without $H^{1}$ regularity in [6], at rate $\sqrt{\epsilon}$ (ignoring boundary conditions and free surface).

Using the expression (2.13), the equation (2.1a) is rewritten as

$$
\rho\left(\partial_{t} \boldsymbol{u}+(\boldsymbol{u} \cdot \nabla) \boldsymbol{u}\right)-\operatorname{div} \boldsymbol{\sigma}_{\epsilon}^{\prime}+\nabla p=\rho \boldsymbol{f} \quad \text { for } t \in(0, T), \vec{X} \in \Omega_{t} .
$$

We set

$$
\begin{aligned}
\boldsymbol{V} & :=\left\{\boldsymbol{v} \in L^{2}\left(0, T ; \boldsymbol{V}_{t}\right) \text { such that } \frac{d \boldsymbol{v}}{d t} \in L^{2}\left(0, T ; \boldsymbol{V}_{t}^{\prime}\right)\right\} \\
M & :=L^{2}\left(0, T ; M_{t}\right)
\end{aligned}
$$

with respectively for the configuration (a) or (b),

(a) $V_{t}:=\left\{\boldsymbol{v} \in H^{1}\left(\Omega_{t}\right)^{2}\right.$ such that $\boldsymbol{v}=\mathbf{0}$ on $\Gamma_{b}, \boldsymbol{v}(\vec{X})=\boldsymbol{v}(\mathcal{T}(\vec{X}))$ for $\left.\vec{X} \in \Gamma_{\ell, t}\right\}$,

(b) $\boldsymbol{V}_{t}:=\left\{\boldsymbol{v} \in H^{1}\left(\Omega_{t}\right)^{2}\right.$ such that $\boldsymbol{v} \cdot \boldsymbol{N}=0$ on $\left.\Gamma_{b, t} \cup \Gamma_{\ell, t}\right\}$,

and $M_{t}=L^{2}\left(\Omega_{t}\right)$. The variational formulation of (2.14), (2.1b), (2.13), (2.11), and (2.5), (2.6) for case (a), (2.7), (2.8) for case (b) consists in finding $(\boldsymbol{u}, p) \in \boldsymbol{V} \times M$ such that the initial condition is satisfied, and for almost all $t \in(0, T)$, and all $(\boldsymbol{v}, q) \in \boldsymbol{V}_{t} \times M_{t}$,

$$
\begin{gathered}
\int_{\Omega_{t}} \rho\left(\partial_{t} \boldsymbol{u}+(\boldsymbol{u} \cdot \nabla) \boldsymbol{u}\right) \cdot \boldsymbol{v}+\int_{\Omega_{t}} 2 \eta D \boldsymbol{u}: D \boldsymbol{v}+\int_{\Omega_{t}} \kappa \frac{D \boldsymbol{u}}{\sqrt{\|D \boldsymbol{u}\|^{2}+\epsilon^{2}}}: D \boldsymbol{v}-\int_{\Omega_{t}} p \operatorname{div} \boldsymbol{v} \\
+\int_{\Gamma_{b, t} \cup \Gamma_{\ell, t}} \mu_{b / \ell} \frac{\boldsymbol{u}_{T} \cdot \boldsymbol{v}}{\sqrt{\left|\boldsymbol{u}_{T}\right|^{2}+\epsilon_{f}^{2}}}\left[p-\sigma_{N}^{\prime}\right]_{+}=\int_{\Omega_{t}} \rho \boldsymbol{f} \cdot \boldsymbol{v}+\int_{\Gamma_{f, t}} \gamma \boldsymbol{v} \cdot \boldsymbol{N} \\
\int_{\Omega_{t}} q \operatorname{div} \boldsymbol{u}=0,
\end{gathered}
$$

where we have also regularized the boundary friction terms with a parameter $\epsilon_{f}>0$. In case (a) no bottom or lateral boundary term should be put in the variational formulation (2.17a) (because of the definition of $\boldsymbol{V}_{t}$ in (2.16a) and of the conditions (2.5), (2.6) that we have to impose), thus we simply set $\mu_{b}=\mu_{\ell}=0$ to make the corresponding terms in (2.17a) vanish.

\section{Discretization of the problem}

\subsection{Variational formulation}

In this section, we describe the numerical method used to solve the problem (2.17). The space discretization is based on mixed finite elements, and the time discretization on a first-order, semi-implicit finite difference scheme.

We consider at each discrete time $t^{n}$, with $t^{0}=0, t^{n+1}=t^{n}+\Delta t_{n}$, with $\Delta t_{n}$ the time step, mixed finite element spaces $\boldsymbol{V}_{h}^{n}$ and $M_{h}^{n}$ spanned by functions defined on a mesh of $\Omega_{h}^{n}$, where $\Omega_{h}^{n}$ is the approximation of the material domain at time $t^{n}$. The approximate velocity and pressure fields are denoted by $\boldsymbol{u}_{h}^{n}$ and $p_{h}^{n}$. We consider the $\mathbb{P}_{2} / \mathbb{P}_{1}$ setting, with triangular Taylor-Hood finite elements. We define the spaces $\mathbb{P}_{2}\left(\Omega_{h}^{n}\right)$ (resp., $\mathbb{P}_{1}\left(\Omega_{h}^{n}\right)$ ) as composed of continuous functions that are piecewise quadratic (resp., affine) on the mesh of $\Omega_{h}^{n}$. In case (a) (periodic flow over inclined bed), we incorporate all the boundary conditions from (2.16a) in $\boldsymbol{V}_{h}^{n}$, whereas in case (b) (granular collapse), the boundary condition in (2.16b) is not enforced explicitly in the definition of $\boldsymbol{V}_{h}^{n}$, but weakly by means of a boundary penalty method. Hence, $\boldsymbol{V}_{h}^{n}$ is a proper subspace of $\mathbb{P}_{2}\left(\Omega_{h}^{n}\right)^{2}$ in case (a) and coincides with $\mathbb{P}_{2}\left(\Omega_{h}^{n}\right)^{2}$ in case (b). Moreover, $M_{h}^{n+1}=\mathbb{P}_{1}\left(\Omega_{h}^{n}\right)$ in both cases. 
At the beginning of the time step from $t^{n}$ to $t^{n+1}$, the discrete velocity field $\boldsymbol{u}_{h}^{n} \in \boldsymbol{V}_{h}^{n}$ (defined on $\Omega_{h}^{n}$ ) is known. We also have at our disposal a second discrete velocity field $\boldsymbol{w}_{h}^{n}$, also defined on $\Omega_{h}^{n}$, and which describes the approximate velocity of the domain $\Omega_{h}^{n}$. The velocity field $\boldsymbol{w}_{h}^{n}$ is built according to the ALE method described in Section 3.2 below. We consider the map

$$
\begin{aligned}
\mathcal{A}_{n, n+1}: \Omega_{h}^{n} & \rightarrow \Omega_{h}^{n+1} \\
\vec{X} & \mapsto \vec{Y}=\vec{X}+\Delta t_{n} \boldsymbol{w}_{h}^{n}(\vec{X}),
\end{aligned}
$$

which defines $\Omega_{h}^{n+1}$ as the image of $\Omega_{h}^{n}$. This map also allows us to obtain a mesh of $\Omega_{h}^{n+1}$ as the image of the mesh of $\Omega_{h}^{n}$, so that $\boldsymbol{w}_{h}^{n}$ is referred to as the mesh velocity.

The discrete velocity and pressure fields $\left(\boldsymbol{u}_{h}^{n+1}, p_{h}^{n+1}\right) \in \boldsymbol{V}_{h}^{n+1} \times M_{h}^{n+1}$ are obtained by means of an iterative algorithm. Denoting with superscript $k$ the variables involved in the iterative algorithm, we introduce the variables $\boldsymbol{u}_{h}^{n+1, k}$ and $p_{h}^{n+1, k}$. We start with $\boldsymbol{u}_{h}^{n+1,0}=\boldsymbol{u}_{h}^{n} \circ \mathcal{A}_{n, n+1}^{-1}, p_{h}^{n+1,0}=p_{h}^{n} \circ \mathcal{A}_{n, n+1}^{-1}$, where $\circ$ denotes the composition of maps. The update algorithm is defined as follows: given $\left(\boldsymbol{u}_{h}^{n+1, k}, p_{h}^{n+1, k}\right)$, find $\left(\boldsymbol{u}_{h}^{n+1, k+1}, p_{h}^{n+1, k+1}\right) \in$ $\boldsymbol{V}_{h}^{n+1} \times M_{h}^{n+1}$ such that for all $\left(\boldsymbol{v}_{h}, q_{h}\right) \in \boldsymbol{V}_{h}^{n+1} \times M_{h}^{n+1}$,

$$
\begin{aligned}
& \int_{\Omega_{h}^{n+1}} \rho\left(\frac{\boldsymbol{u}_{h}^{n+1, k+1}-\boldsymbol{u}_{h}^{n} \circ \mathcal{A}_{n, n+1}^{-1}}{\Delta t_{n}}+\left[\left(\boldsymbol{u}_{h}^{n+1, k}-\boldsymbol{w}_{h}^{n} \circ \mathcal{A}_{n, n+1}^{-1}\right) \cdot \nabla\right] \boldsymbol{u}_{h}^{n+1, k+1}\right) \cdot \boldsymbol{v}_{h} \\
& +\int_{\Omega_{h}^{n+1}}\left(2 \eta+\frac{\kappa_{h}^{n+1, k}}{\sqrt{\left\|D \boldsymbol{u}_{h}^{n+1, k}\right\|^{2}+\epsilon^{2}}}\right) D \boldsymbol{u}_{h}^{n+1, k+1}: D \boldsymbol{v}_{h} \\
& -\int_{\Omega_{h}^{n+1}} p_{h}^{n+1, k+1} \operatorname{div} \boldsymbol{v}_{h}+\int_{\Gamma_{b, h}^{n+1} \cup \Gamma_{\ell, h}^{n+1}} \mu_{b / \ell} \frac{\boldsymbol{u}_{T, h}^{n+1, k+1} \cdot \boldsymbol{v}_{h}}{\sqrt{\left|\boldsymbol{u}_{T, h}^{n+1, k}\right|^{2}+\epsilon_{f}^{2}}}\left[p_{h}^{n+1, k}-\sigma^{\prime n+1, k}\right]_{+} \\
& +\int_{\Gamma_{b, h}^{n+1} \cup \Gamma_{\ell, h}^{n+1}} \xi\left(\boldsymbol{u}_{h}^{n+1, k+1} \cdot \boldsymbol{N}\right)\left(\boldsymbol{v}_{h} \cdot \boldsymbol{N}\right)=\int_{\Omega_{h}^{n+1}} \rho \boldsymbol{f}^{n+1} \cdot \boldsymbol{v}_{h}+\int_{\Gamma_{f, h}^{n+1}} \gamma \boldsymbol{v}_{h} \cdot \boldsymbol{N}, \\
& \int_{\Omega_{h}^{n+1}} q_{h} \operatorname{div} \boldsymbol{u}_{h}^{n+1, k+1}=0,
\end{aligned}
$$

where $\kappa_{h}^{n+1, k}=\sqrt{2} \mu_{s}\left[p_{h}^{n+1, k}\right]_{+}$, and $\xi \gg 1$ is a penalty parameter used in case (b) (we set $\xi=0$ in case (a)). In our simulations, we take $\xi=10^{12}$. The iteration stopping criterion is $\left\|\boldsymbol{u}_{h}^{n+1, k}-\boldsymbol{u}_{h}^{n+1, k-1}\right\|_{L^{2}} /\left\|\boldsymbol{u}_{h}^{n+1, k-1}\right\|_{L^{2}}<\varepsilon_{\text {iter }}$. It is also possible to impose an upper bound $k_{\max }$ on the number of iterations.

The time step is taken constant, except during the starting stage. Indeed, since the initial velocity vanishes, the problem is quite stiff at the beginning, because the static-flowing interface has to be determined. Therefore, we take initially a quite small time step $\Delta t_{\text {init }}$, it is then slowly increased by applying the formula $\Delta t_{n+1}=1.1 \Delta t_{n}$, until it reaches a desired value $\Delta t_{\max }$. Afterwards it is taken constant $\Delta t_{n}=\Delta t_{\max }$. Similarly, we start with $\varepsilon_{\text {iter }}$ quite small and increase it slowly to a prescribed value.

\subsection{Displacement of the domain}

The mesh velocity $\boldsymbol{w}_{h}^{n}$ used for computing the new mesh of $\Omega_{h}^{n+1}$ by (3.1) needs to be consistent with the kinematic boundary condition (2.12), i.e., it must satisfy $\boldsymbol{w}_{h}^{n} \cdot \boldsymbol{N}=\boldsymbol{u}_{h}^{n} \cdot \boldsymbol{N}$ on $\Gamma_{f, h}^{n}$. It must also satisfy $\boldsymbol{w}_{h}^{n} \cdot \boldsymbol{N}=0$ on the static boundaries. In order to define $\boldsymbol{w}_{h}^{n}$, we extend suitable boundary values by solving an elliptic problem inside $\Omega_{h}^{n}$,

$$
-\operatorname{div}\left(D \boldsymbol{w}_{h}^{n}\right)=0 \quad \text { in } \Omega_{h}^{n}
$$


with in case (a)

$$
\begin{aligned}
& \left(\boldsymbol{w}_{h}^{n}-\boldsymbol{u}_{h}^{n}\right) \cdot \boldsymbol{N}=0 \quad \text { on } \Gamma_{b, h} \cup \Gamma_{f, h}^{n}, \\
& \boldsymbol{w}_{h}^{n} \cdot \boldsymbol{N}=0 \quad \text { on } \Gamma_{\ell, h}^{n} \cup \Gamma_{r, h}^{n}, \\
& \left(D \boldsymbol{w}_{h}^{n} \boldsymbol{N}\right)_{T}=0 \quad \text { on } \Gamma_{h}^{n} \text {, }
\end{aligned}
$$

and in case (b)

$$
\begin{aligned}
\left(\boldsymbol{w}_{h}^{n}-\boldsymbol{u}_{h}^{n}\right) \cdot \boldsymbol{N}=0 & \text { on } \Gamma_{h}^{n}, \\
\left(D \boldsymbol{w}_{h}^{n} \boldsymbol{N}\right)_{T}=0 & \text { on } \Gamma_{h}^{n},
\end{aligned}
$$

where $\left(D \boldsymbol{w}_{h}^{n} \boldsymbol{N}\right)_{T}$ is the tangential part of the vector $D \boldsymbol{w}_{h}^{n} \boldsymbol{N}$. In the formulations (3.3) with (3.4) or (3.5), the tangential component of $\boldsymbol{w}_{h}^{n}$ on the boundary is not imposed, but is free to adapt (we expect that the resulting solution $\boldsymbol{w}_{h}^{n}$ is smoother). This problem is discretized using the same finite element space as for the discrete velocity $\boldsymbol{u}_{h}^{n}$, with a boundary penalty method. Thus, we look for $\boldsymbol{w}_{h}^{n} \in \mathbb{P}_{2}\left(\Omega_{h}^{n}\right)^{2}$ such that for all $\boldsymbol{v}_{h} \in \mathbb{P}_{2}\left(\Omega_{h}^{n}\right)^{2}$,

$$
\int_{\Omega_{h}^{n}} D \boldsymbol{w}_{h}^{n}: D \boldsymbol{v}_{h}+\int_{\Gamma_{h}^{n}} \hat{\xi}\left(\boldsymbol{w}_{h}^{n} \cdot \boldsymbol{N}-\boldsymbol{u}_{h}^{n} \cdot \boldsymbol{N}\right)\left(\boldsymbol{v}_{h} \cdot \boldsymbol{N}\right)=0
$$

where $\hat{\xi} \gg 1$ is a penalty parameter (set to $10^{12}$ in our simulations). This is valid for case (b). For case (a) we replace in (3.6) $\boldsymbol{u}_{h}^{n} \cdot \boldsymbol{N}$ by $\boldsymbol{u}_{h}^{n} \cdot \boldsymbol{N} \mathbb{I}_{\Gamma_{b, h} \cup \Gamma_{f, h}^{n}}$.

Although the formulation (3.6) worked satisfactorily in our simulations, it is worthwhile to mention that it does not provide an optimal distribution of points along the boundary as the mesh evolves in time. This difficulty is linked to the enforcement of the boundary condition on the normal velocity component using Lagrange finite elements in a domain with an irregular boundary where the outward normal changes direction from one boundary edge to the neighboring one. Indeed, taking $\boldsymbol{v}=\boldsymbol{w}_{h}^{n}-\boldsymbol{u}_{h}^{n}$ in (3.6) imposes $\boldsymbol{w}_{h}^{n} \cdot \boldsymbol{N}-\boldsymbol{u}_{h}^{n} \cdot \boldsymbol{N}=0$ everywhere on the boundary, which yields $\boldsymbol{w}_{h}^{n}=\boldsymbol{u}_{h}^{n}$ (i.e., pure Lagrangian transport) at every boundary point where $\boldsymbol{N}$ is discontinuous. This contradicts the previously postulated freedom of the tangential component of $\boldsymbol{w}_{h}^{n}$. This difficulty is well-known, e.g., in electromagnetism applications where edge finite elements are preferable to Lagrange finite elements in some situations, see [12] and [24, p. 97]. Another work-around, still using Lagrange finite elements, is to enforce the boundary condition by means of a Lagrange multiplier distributed in $\Omega_{h}^{n}$, see, e.g., [34], leading to the following formulation: find $\left(\boldsymbol{w}_{h}^{n}, \widetilde{p}_{h}^{n}\right) \in \mathbb{P}_{2}\left(\Omega_{h}^{n}\right)^{2} \times \mathbb{P}_{2}\left(\Omega_{h}^{n}\right)$ such that for all $\left(\boldsymbol{v}_{h}, q_{h}\right) \in \mathbb{P}_{2}\left(\Omega_{h}^{n}\right)^{2} \times \mathbb{P}_{2}\left(\Omega_{h}^{n}\right)$,

$$
\int_{\Omega_{h}^{n}} D \boldsymbol{w}_{h}^{n}: D \boldsymbol{v}_{h}+\hat{\xi}^{-1} \int_{\Omega_{h}^{n}} \widetilde{p}_{h}^{n} q_{h}+\int_{\Gamma_{h}^{n}} q_{h}\left(\boldsymbol{w}_{h}^{n}-\boldsymbol{u}_{h}^{n}\right) \cdot \boldsymbol{N}+\int_{\Gamma_{h}^{n}} \widetilde{p}_{h}^{n}\left(\boldsymbol{v}_{h} \cdot \boldsymbol{N}\right)=0 .
$$

This formulation enables the boundary points to be better distributed; note, in particular, that the continuity requirement on the test function $q_{h}$ prevents us from taking $q_{h}=\left(\boldsymbol{w}_{h}^{n}-\right.$ $\left.\boldsymbol{u}_{h}^{n}\right) \cdot \boldsymbol{N}$ in (3.7).

\subsection{Update algorithm and free surface deposition onto the bottom}

We write down here the steps of the algorithm, with in particular a step of deposition of the free surfrace onto the bottom. At the start of step $n, \Omega_{h}^{n}, \boldsymbol{u}_{h}^{n}, p_{h}^{n}$ are known.

1) We compute $\boldsymbol{w}_{h}^{n}$ by solving (3.6) in case (b), or its modification with $\boldsymbol{u}_{h}^{n} \cdot \boldsymbol{N} \mathbb{1}_{\Gamma_{b, h} \cup \Gamma_{f, h}^{n}}$ in case (a).

2) We move the nodes of the mesh according to $\mathcal{A}_{n, n+1}$ defined by (3.1). Doing this, in case (b) with rigid bed we may need to limit the time step so that the free surface nodes do not cross the bottom or the left wall of the domain. For this we apply the free surface deposition procedure described in the caption of Figure 2. Thus, we obtain $\Omega_{h}^{n+1}$. 
3) Finally, we compute $\left(\boldsymbol{u}_{h}^{n+1}, p_{h}^{n+1}\right)$ on $\Omega_{h}^{n+1}$ with the iterative formulation (3.2).
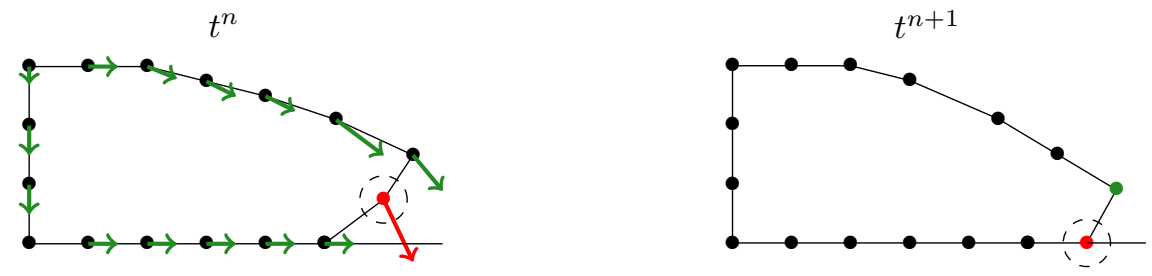

Figure 2: Schematic view of the free surface deposition procedure in the case when a free surface node goes to the bottom for the configuration (b) with rigid bed. (Left) Dots represent the boundary nodes at time $t^{n}$. The arrows represent the displacement $\Delta t \boldsymbol{w}_{h}^{n}$ that is used in (3.1), before limitation of the time step. (Right) New position of the nodes at time $t^{n+1}$. The time step has been diminished so that no boundary node crosses the bottom. In the illustrated situation, one node that was on the free surface at time $t^{n}$ is now at the bottom boundary. One boundary edge that was part of the free surface is now part of the bottom (the nature of boundary conditions applied to it has to change accordingly). In our simulations of configuration (b) with rigid bed, the effective change of nature of a part of the free surface occurs at most twice between initial and final time.

The code is written in FreeFem ++ , see [34]. To illustrate the movement of the mesh in a practical situation (the test case of granular collapse of a mass with an initial trapezoidal shape described in Section 5), we plot on Figure 3 the initial and final meshes obtained in our simulations. Two boundary edges that were initially on the free surface have moved to the bottom. As commented above, the points on the free surface have moved by pure Lagrangian transport.
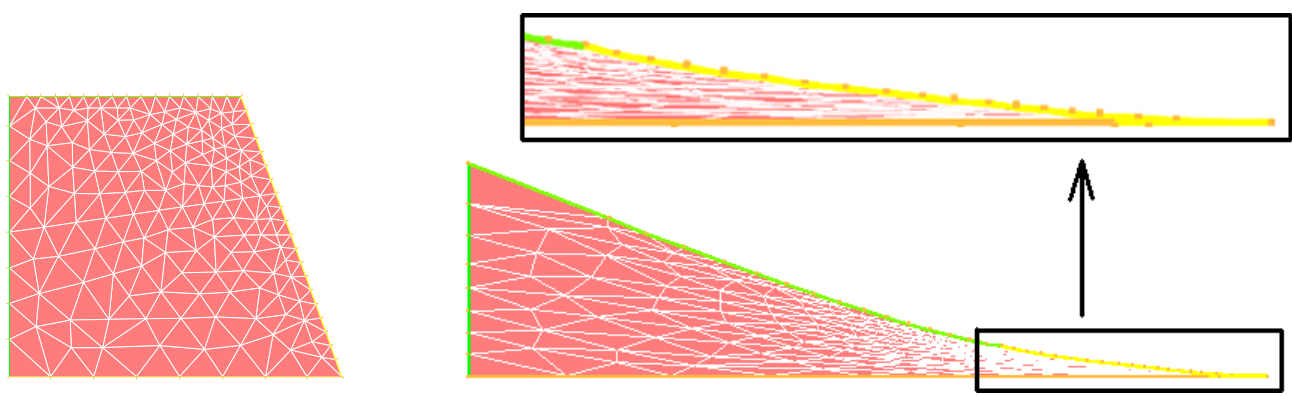

Figure 3: Initial (left) and final (right) meshes for the granular collapse with trapezoidal geometry considered in Section 5, with regularization parameter $\epsilon=10^{-6} \mathrm{~s}^{-1}$. The upper right plot is a zoom of the tip of the granular mass, that shows the location of the boundary nodes. The different colors of boundary edges correspond to the different parts of the initial boundary.

\subsection{Surface tension}

In the case of granular collapse with erodible bed, the evolution of the domain can lead to a folding of the free surface over itself, which is related to the fact that the flowing material has the tendency to cover the bed, as shown on Figure 4 (right). This is indeed the same physical phenomenon that is observed on rigid bottom, but the mathematical difficulty is of different nature, and needs a different treatment than the one used on rigid bottom (free surface deposition algorithm of Subsection 3.3). Without particular treatment the ALE method 
cannot work on erodible bed, because of the folding. In order to address this difficulty, we add a numerical surface tension that has the effect of replacing a folded surface by a straight one. We thus impose (2.11) at the free surface with parameter $\gamma$ such that

$$
\gamma=\gamma_{0} \mathcal{C}
$$

where $\gamma_{0} \geq 0$ is a position-dependent, small parameter of the order of the mesh size, and $\mathcal{C}$ is the local curvature of the free surface, with negative sign in the case of a locally convex domain $\Omega_{t}$ and positive otherwise.

The local curvature is given by $\mathcal{C}=d \theta / d s$, with $\theta$ the angle of the tangent to the boundary with respect to a fixed direction and $s$ the curvilinear coordinate oriented clockwise. Referring to Figure 4, we compute the angular variation $\delta \theta_{i}$ between two edges of the free surface connected by the node $N_{i}$ as follows:

$$
\delta \theta_{i}=2 \arctan \left(\frac{\vec{R}_{i+1} \cdot \vec{R}_{i}^{\perp}}{\left|\vec{R}_{i+1}\right|\left|\vec{R}_{i}\right|+\vec{R}_{i+1} \cdot \vec{R}_{i}}\right),
$$

where $\vec{R}_{i}=\vec{N}_{i-1} N_{i}$. An approximation of the curvature at $N_{i}$ is thus given by

$$
\mathcal{C}_{i}=\frac{\delta \theta_{i}}{\left(\left|\vec{R}_{i+1}\right|+\left|\vec{R}_{i}\right|\right) / 2} .
$$

We denote by $i_{c}$ the critical node index where the curvature is the largest. We apply locally the surface tension around the critical node $i_{c}$ by setting

$$
\gamma_{0, i}=\bar{\gamma}_{0}\left(\max \left\{0,1-\left(\frac{i-i_{c}}{\delta}\right)^{2}\right\}\right)^{2},
$$

where $\delta$ is a positive coefficient that determines the extension of the surface tension application. In our simulations, we set $\delta=16$, and we take $\bar{\gamma}_{0}$ as the order of magnitude of the pressure in the first cells close to the free surface, times the mesh size. The effect of this
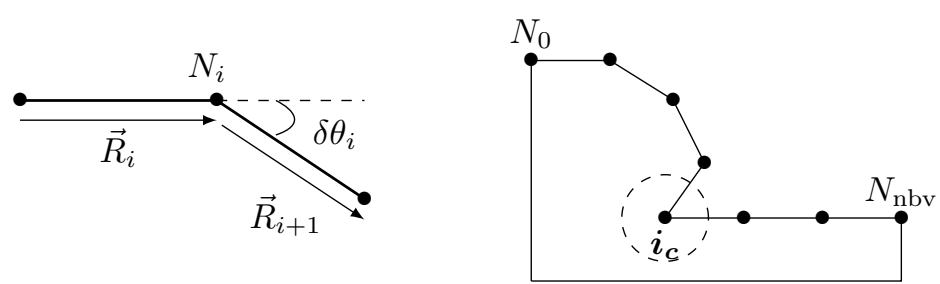

Figure 4: Boundary nodes $N_{i}$ and boundary vectors $\vec{R}_{i}$ and $\vec{R}_{i+1}$.

numerical surface tension is to flatten the free surface when it becomes too much curved. We have checked that its application does not alter the numerical results when it is not needed, i.e., when the free surface is not too much curved. For instance, in the case of granular collapse of a trapezoidal mass over a rigid bed, we obtain very similar profiles for the free surface if we apply the surface tension or not, as shown on Figure 5. 

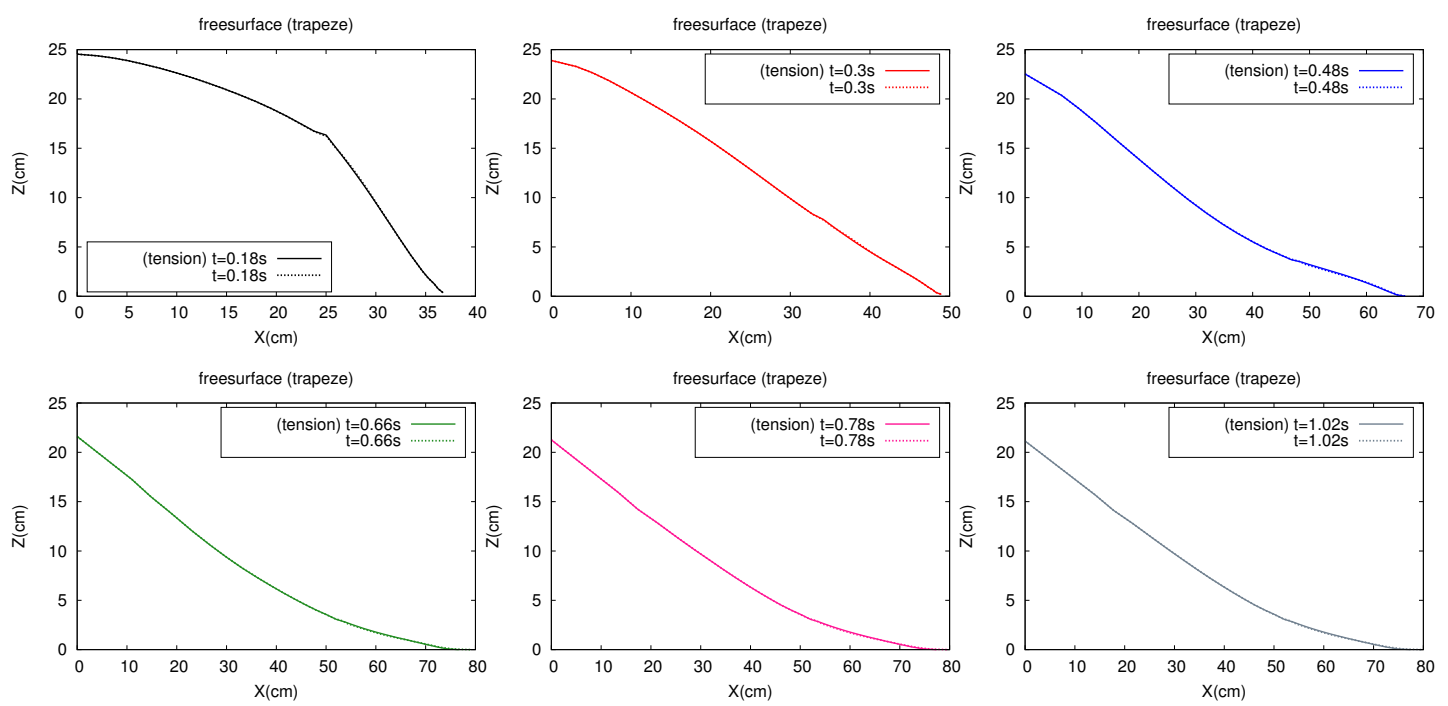

Figure 5: Comparison of the evolution of the free surface for a trapezoidal granular mass over a rigid bed, at times $t=0.18 \mathrm{~s}, t=0.3 \mathrm{~s}, t=0.48 \mathrm{~s}, t=0.66 \mathrm{~s}, t=0.78 \mathrm{~s}, t=1.02 \mathrm{~s}$, between the regularization method with surface tension (full lines) and the regularization method without surface tension (dotted lines). The full and dotted lines are indeed almost indistinguishable.

\section{Simple shear plug flow over an inclined bed}

The goal of this section is to evaluate the accuracy of our two-dimensional regularization method. We consider a test case involving a one-dimensional solution, depending only on the normal variable $Z$, that has been described in [8]. The solution has velocity $\boldsymbol{u}=(U, 0)$, where the longitudinal velocity $U(t, Z)$ is defined for $0<Z<h$ (the height of the domain $h$ being constant), vanishes for $0<Z<b(t)$, and solves for $b(t)<Z<h$

$$
\begin{aligned}
& \partial_{t} U+S-\partial_{Z}\left(\nu \partial_{Z} U\right)=0 \quad \text { for all } Z \in(b(t), h), \\
& U=0 \quad \text { at } Z=b(t), \\
& \nu \partial_{Z} U=0 \quad \text { at } Z=b(t), \\
& \nu \partial_{Z} U=0 \quad \text { at } Z=h,
\end{aligned}
$$

with $S$ a constant source term given by

$$
S=g \cos \theta\left(\mu_{s}-\tan \theta\right),
$$

where $\mu_{s}$ comes from (2.4), $\mu_{s}=\tan \delta$ with $\delta$ the friction angle, $\theta>0$ is the angle of the inclined plane as shown on Figure 1a, and $\nu=\eta / \rho$ is the kinematic viscosity. The static/flowing interface $b(t)$ is an unknown in the problem (4.1). According to [8, Proposition 2.2], if $\delta \geq \theta$ and $\partial_{Z} U>0$ for $Z \in(b(t), h)$, then $\boldsymbol{u}=(U, 0)$ is a solution to (2.1), (2.2), (2.4) that satisfies the boundary conditions of configuration (a). Moreover, the pressure is hydrostatic, $p=\rho g \cos \theta(h-Z)$, and the domain does not depend on time.

It is shown in $[47,48]$ that the dynamical behavior of the system $(4.1),(4.2)$ is as follows. The static/flowing interface position $b(t)$ first moves down as a consequence of viscosity until a time $t^{\mathrm{c}}$, and attains a minimal value $b^{\text {min }}$ (starting phase with erosion). Then $b(t)$ moves up as a consequence of friction, and (if $h$ is sufficiently large) reaches an asymptotic regime with upward velocity $\dot{b}^{\infty}$ (stopping phase with deposition), before fully stopping when attaining $h$.

We compute accurately the one-dimensional solution to (4.1) by one of the two methods described in $[47,48]$. Then we solve the full two-dimensional problem by the method 
described in Section 3 for configuration (a). In order to evaluate the error between the one-dimensional profile and the longitudinal component of the two-dimensional velocity, we extend the one-dimensional solution to the two-dimensional mesh, by interpolating the onedimensional profile in $Z$ in each cell. We compute the relative error on the velocity, in the $L^{2}$-norm, and we denote it by $e_{L^{2}}(\boldsymbol{u})$.

The test is performed within the rectangular domain $\Omega=[0, l] \times[0, h]$ with length $l=1.5 \mathrm{~m}$ and height $h=6 \mathrm{~m}$ (see Figure 1a). The bottom is an inclined plane with angle $\theta=1^{\circ}$. The friction angle is taken so that $\tan \delta=\tan \theta+10^{-2}$. Initially, the longitudinal velocity has a piecewise affine profile $U^{0}(Z)=\alpha_{1}\left[Z-b^{0}\right]_{+}$, with $\alpha_{1}=1 \mathrm{~s}^{-1}$ and $b^{0}$ the initial thickness of the solid layer, chosen to be $b^{0}=3.5 \mathrm{~m}$. We consider the evolution of the material up to the time $T=0.2 \mathrm{~s}$, a time for which the simulated mass is not yet at rest, making it possible to evaluate the numerical velocity profile. We compute the one-dimensional solution with a cell length of $10^{-4} \mathrm{~m}$ and an initial time step $\Delta t^{0}=10^{-4} \mathrm{~s}$. For the two-dimensional problem, we consider a structured rectangular mesh with cell length $\Delta Z$. We have verified the convergence of our numerical solutions by halving the mesh size and the time step $\Delta t$. In the semi-implicit resolution of the two-dimensional problem, we use an iteration stopping tolerance $\varepsilon_{\text {iter }}=10^{-7}$. We compute the solution for a viscosity $\nu=1 \mathrm{~m}^{2} / \mathrm{s}$. Finally we take several regularization parameters, $\epsilon=10^{-2} \mathrm{~S}^{-1}, 10^{-4} \mathrm{~s}^{-1}$, and $10^{-6} \mathrm{~s}^{-1}$, so as to study the influence of the choice of $\epsilon$.

\begin{tabular}{|c|c|c|c|}
\hline$\epsilon=10^{-2} \mathrm{~s}^{-1}$ & $\Delta Z=2.5 \mathrm{e}-01 \mathrm{~m}$ & $\Delta Z=1.25 \mathrm{e}-01 \mathrm{~m}$ & $\Delta Z=6.25 \mathrm{e}-02 \mathrm{~m}$ \\
\hline$\Delta t=2.5 \mathrm{e}-02 \mathrm{~s}$ & $3.02 \mathrm{e}-02$ & $2.99 \mathrm{e}-02$ & $2.99 \mathrm{e}-02$ \\
\hline$\Delta t=1.25 \mathrm{e}-02 \mathrm{~s}$ & $2.75 \mathrm{e}-02$ & $2.73 \mathrm{e}-02$ & $2.73 \mathrm{e}-02$ \\
\hline$\Delta t=6.25 \mathrm{e}-03 \mathrm{~s}$ & $2.65 \mathrm{e}-02$ & $2.64 \mathrm{e}-02$ & $2.64 \mathrm{e}-02$ \\
\hline$\Delta t=3.12 \mathrm{e}-03 \mathrm{~s}$ & $2.61 \mathrm{e}-02$ & $2.60 \mathrm{e}-02$ & $2.60 \mathrm{e}-02$ \\
\hline$\Delta t=1.56 \mathrm{e}-03 \mathrm{~s}$ & $2.59 \mathrm{e}-02$ & $2.59 \mathrm{e}-02$ & $2.59 \mathrm{e}-02$ \\
\hline$\Delta t=7.78 \mathrm{e}-04 \mathrm{~s}$ & $2.59 \mathrm{e}-02$ & $2.58 \mathrm{e}-02$ & $2.58 \mathrm{e}-02$ \\
\hline
\end{tabular}

\begin{tabular}{|c|c|c|c|}
\hline$\epsilon=10^{-4} \mathrm{~s}^{-1}$ & $\Delta Z=2.5 \mathrm{e}-01 \mathrm{~m}$ & $\Delta Z=1.25 \mathrm{e}-01 \mathrm{~m}$ & $\Delta Z=6.25 \mathrm{e}-02 \mathrm{~m}$ \\
\hline$\Delta t=2.5 \mathrm{e}-02 \mathrm{~s}$ & $1.26 \mathrm{e}-02$ & $1.19 \mathrm{e}-02$ & $1.17 \mathrm{e}-02$ \\
\hline$\Delta t=1.25 \mathrm{e}-02 \mathrm{~s}$ & $6.87 \mathrm{e}-03$ & $6.18 \mathrm{e}-03$ & $5.99 \mathrm{e}-03$ \\
\hline$\Delta t=6.25 \mathrm{e}-03 \mathrm{~s}$ & $4.01 \mathrm{e}-03$ & $3.28 \mathrm{e}-03$ & $3.11 \mathrm{e}-03$ \\
\hline$\Delta t=3.12 \mathrm{e}-03 \mathrm{~s}$ & $2.60 \mathrm{e}-03$ & $1.85 \mathrm{e}-03$ & $1.67 \mathrm{e}-03$ \\
\hline$\Delta t=1.56 \mathrm{e}-03 \mathrm{~s}$ & $1.89 \mathrm{e}-03$ & $1.16 \mathrm{e}-03$ & $1.01 \mathrm{e}-03$ \\
\hline$\Delta t=7.78 \mathrm{e}-04 \mathrm{~s}$ & $1.55 \mathrm{e}-03$ & $8.28 \mathrm{e}-04$ & $7.13 \mathrm{e}-04$ \\
\hline
\end{tabular}

\begin{tabular}{|c|c|c|c|}
\hline$\epsilon=10^{-6} \mathrm{~s}^{-1}$ & $\Delta Z=2.5 \mathrm{e}-01 \mathrm{~m}$ & $\Delta Z=1.25 \mathrm{e}-01 \mathrm{~m}$ & $\Delta Z=6.25 \mathrm{e}-02 \mathrm{~m}$ \\
\hline$\Delta t=2.5 \mathrm{e}-02 \mathrm{~s}$ & $1.25 \mathrm{e}-02$ & $1.18 \mathrm{e}-02$ & $1.16 \mathrm{e}-02$ \\
\hline$\Delta t=1.25 \mathrm{e}-02 \mathrm{~s}$ & $6.77 \mathrm{e}-03$ & $6.06 \mathrm{e}-03$ & $5.87 \mathrm{e}-03$ \\
\hline$\Delta t=6.25 \mathrm{e}-03 \mathrm{~s}$ & $3.89 \mathrm{e}-03$ & $3.15 \mathrm{e}-03$ & $2.96 \mathrm{e}-03$ \\
\hline$\Delta t=3.12 \mathrm{e}-03 \mathrm{~s}$ & $2.47 \mathrm{e}-03$ & $1.69 \mathrm{e}-03$ & $1.50 \mathrm{e}-03$ \\
\hline$\Delta t=1.56 \mathrm{e}-03 \mathrm{~s}$ & $1.75 \mathrm{e}-03$ & $9.69 \mathrm{e}-04$ & $7.70 \mathrm{e}-04$ \\
\hline$\Delta t=7.78 \mathrm{e}-04 \mathrm{~s}$ & $1.40 \mathrm{e}-03$ & $6.15 \mathrm{e}-04$ & $4.13 \mathrm{e}-04$ \\
\hline
\end{tabular}

Table 1: Relative error $e_{L^{2}}(\boldsymbol{u})$ without moving mesh for plug flow over an inclined bed for various values of the time step $\Delta t$, mesh size $\Delta Z$, and regularization parameter $\epsilon$.

We first consider the relative error $e_{L^{2}}(\boldsymbol{u})$ without moving the mesh, i.e., setting the mesh velocity to $\boldsymbol{w}=\mathbf{0}$ in (3.1) and (3.2a). This error is reported in Table 1 for $\epsilon=10^{-2} \mathrm{~s}^{-1}$ (top table), $\epsilon=10^{-4} \mathrm{~s}^{-1}$ (middle table), and $\epsilon=10^{-6} \mathrm{~S}^{-1}$ (bottom table). For $\epsilon=10^{-2} \mathrm{~s}^{-1}$, we can see that the regularization error dominates since diminishing the values of $\Delta t$ or $\Delta Z$ 
does not improve much the accuracy. For $\epsilon=10^{-4} \mathrm{~s}^{-1}$, we can see that for the first four time steps, the error in time dominates with a first-order convergence. Then, the regularization error dominates and the total error stagnates at about $10^{-3}$. For $\epsilon=10^{-6} \mathrm{~s}-1$, we observe a first-order convergence in time for the first time step, and then the errors in space and time are balanced. This means that the regularization parameter $\epsilon=10^{-6} \mathrm{~s}^{-1}$ represents a suitable choice, since it allows us to obtain a regularization error that is negligible with respect to, or of the same order as, the discretization error.

Next we perform the same comparison, but on a moving mesh with a vertical mesh velocity $\boldsymbol{w}=(0, w)$, as explained in [47], and $\epsilon=10^{-6} \mathrm{~s}^{-1}$. The results are presented in Table 2 where we report the relative error $e_{L^{2}}(\boldsymbol{u})$ along with the relative error $e(\Omega)$ on the volume of the domain. Table 2 shows that volume conservation is ensured with an accuracy of order $10^{-5}$. The relative error $e_{L^{2}}(\boldsymbol{u})$ is only slightly affected by the mesh movement since we obtain values very close to those of the two last lines of Table 1 . Notice that for $\epsilon=10^{-8} \mathrm{~S}^{-1}$, we obtain the same results as for $\epsilon=10^{-6} \mathrm{~s}^{-1}$.

\begin{tabular}{|c|c|c|c|c|}
\hline$\epsilon=10^{-6} \mathrm{~s}^{-1}$ & & $\Delta Z=2.5 \mathrm{e}-01 \mathrm{~m}$ & $\Delta Z=1.25 \mathrm{e}-01 \mathrm{~m}$ & $\Delta Z=6.25 \mathrm{e}-02 \mathrm{~m}$ \\
\hline$\Delta t=1.56 \mathrm{e}-03 \mathrm{~s}$ & $e_{L^{2}}(\boldsymbol{u})$ & $1.79 \mathrm{e}-03$ & $9.73 \mathrm{e}-04$ & $7.71 \mathrm{e}-04$ \\
\hline & $e(\Omega)$ & $6.62 \mathrm{e}-05$ & $2.10 \mathrm{e}-05$ & $7.68 \mathrm{e}-06$ \\
\hline$\Delta t=7.78 \mathrm{e}-04 \mathrm{~s}$ & $e_{L^{2}}(\boldsymbol{u})$ & $1.45 \mathrm{e}-03$ & $6.21 \mathrm{e}-04$ & $4.13 \mathrm{e}-04$ \\
\hline & $e(\Omega)$ & $6.62 \mathrm{e}-05$ & $2.11 \mathrm{e}-05$ & $7.69 \mathrm{e}-06$ \\
\hline
\end{tabular}

Table 2: Relative error $e_{L^{2}}(\boldsymbol{u})$ and relative error $e(\Omega)$ on volume conservation with moving mesh for plug flow over an inclined bed for various values of the time step $\Delta t$, mesh size $\Delta Z$, and regularization parameter set to $\epsilon=10^{-6} \mathrm{~s}^{-1}$.

Finally, we observe that the total number of iterations used in the numerical resolution for $\Delta Z=1.25 \times 10^{-1} \mathrm{~m}$ and $\Delta t=3.12 \times 10^{-3} \mathrm{~s}$ are $824,2958,5086$, and 5272 for $\epsilon=10^{-2} \mathrm{~s}^{-1}$, $10^{-4} \mathrm{~S}^{-1}, 10^{-6} \mathrm{~S}^{-1}$, and $10^{-8} \mathrm{~s}^{-1}$, respectively. Thus, this number swiftly increases as $\epsilon$ is decreased in the range from $10^{-2} \mathrm{~s}^{-1}$ to $10^{-6} \mathrm{~s}^{-1}$, while it increases only slightly from $10^{-6} \mathrm{~s}^{-1}$ to $10^{-8} \mathrm{~S}^{-1}$.

The conclusion of our numerical tests on the simple shear plug flow configuration is that the regularization method can deliver accurate results in this setting, and that there is an optimal choice of the regularization parameter $\epsilon$ and of the time step $\Delta t$ in order to minimize the cost for a given accuracy associated with the grid size. Taking $\epsilon$ too small does not, however, increase too much the computational cost. We propose some formulas to compute $\epsilon$ and $\Delta t$ in Subsection 5.1 in the more general setting of the collapse of granular columns.

\section{Granular collapse over rigid or erodible beds}

In this section we apply our method to the classical granular collapse problem. In order to assess the accuracy and physical relevance of our method, we proceed in two steps. We first make a comparison with numerical results obtained with an augmented Lagrangian method described in $[35,36]$. Then we compare our numerical results to those obtained with laboratory experiments, see [25, 51]. These comparisons are made for initial and boundary conditions corresponding to the configuration (b) described in Subsection 2.1. We study the evolution of the free surface, i.e., the thickness profile, the velocity profiles, and the position of the static/flowing interface. 


\subsection{Configuration and choice of parameters}

\subsubsection{Setup and physical parameters}

Different geometries are considered (Figure 6). We study the case of a trapezoidal and a rectangular domain (Figure 6-(I)-(II)) released from rest over a rigid (non-erodible) horizontal bed, and the case of a trapezoidal domain released on a thin erodible bed covering the horizontal bottom (Figure 6-(III)). Beyond its physical interest, this last configuration has been chosen because folding of the granular mass near the front is observed experimentally, and is difficult to handle numerically as discussed above. We denote by $l_{0}, h_{0}$, and $\alpha_{0}$ the initial dimensions of the domain and the slope angle of the released mass, respectively, and by $h_{e}$ the thickness of the erodible bed.

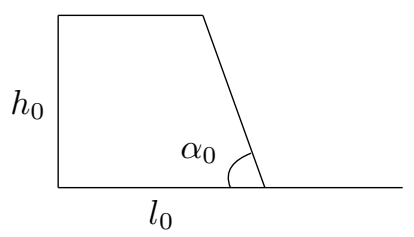

(I) trapeze - rigid bed

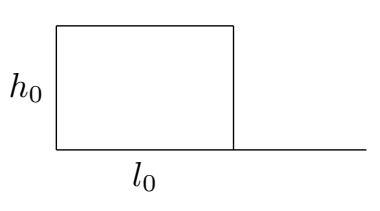

(II) rectangle - rigid bed

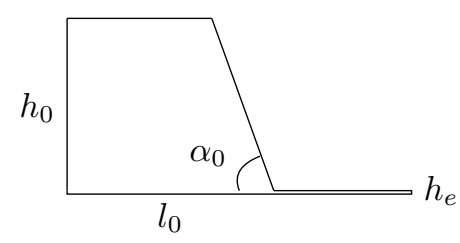

(III) trapeze - erodible bed

Figure 6: (I) Trapezoidal and (II) rectangular mass released over a rigid bed, and (III) trapezoidal mass released over an erodible bed. In all cases the bed is horizontal.

According to the experiments, the geometrical parameters are chosen as: (I) $l_{0}=29.7 \mathrm{~cm}$, $h_{0}=25 \mathrm{~cm}, \alpha_{0}=70^{\circ}$, (II) $l_{0}=20 \mathrm{~cm}, h_{0}=14 \mathrm{~cm}$, and (III) $l_{0}=80 \mathrm{~cm}, h_{0}=25.5 \mathrm{~cm}$, $h_{e}=5 \mathrm{~mm}$. Note that the erodible bed is very thin $\left(h_{e} \ll h_{0}\right)$. Along with gravity set to $g=9.81 \mathrm{~m} . \mathrm{s}^{-2}$, we consider the following rheological parameters: dynamic viscosity $\eta=1 \mathrm{~Pa} . \mathrm{s}$ (this value is chosen in order to represent the effective viscosity that would come out from a $\mu(I)$ rheology in the case of the considered granular collapses, see [35]), mass density $\rho=1550 \mathrm{~kg} \cdot \mathrm{m}^{-3}$, friction angle $\delta=25.5^{\circ}$, friction coefficient of the bottom $\mu_{b}=\tan \left(25.5^{\circ}\right)$, friction coefficient along the left wall $\mu_{l}=\tan \left(10.2^{\circ}\right.$ ) (see [35] for more details about the choice of the parameters value).

\subsubsection{Numerical parameters}

The numerical parameters are set as follows. The regularization parameters are taken to be $\epsilon=10^{-6} \mathrm{~s}^{-1}, \epsilon_{f}=10^{-6} \mathrm{~m} . \mathrm{s}^{-1}$, the mesh size is about $\Delta X \sim 10^{-2} \mathrm{~m}$, with (I) 326 triangles, see Figure 3, (II) 308 triangles, (III) 483 triangles. The time step is $\Delta t_{\max }=10^{-4} \mathrm{~s}$ with $\Delta t_{\text {init }}=10^{-8} \mathrm{~S}$ (see the last paragraph of Subsection 3.1), and the iteration stopping tolerance is $\varepsilon_{\text {iter }}=10^{-4}$.

The choice of the time step $\Delta t_{\max }$ and of the regularization parameter $\epsilon$ deserves some comments. The time step $\Delta t_{\max }$ has to be chosen so as to produce sufficiently small time discretization errors in computing the interface position and so that the iterative algorithm in the semi-implicit time stepping converges. Reasonable bounds on the time step can be inferred from (2.14) and (2.13). A first bound, related to an advective time scale, is $\Delta t \lesssim$ $\|\nabla \boldsymbol{u}\|^{-1}$. A second one, related to the viscosity, is $\Delta t \lesssim \rho \Delta X^{2} / \eta$. A third one, related to the yield stress (and taking into account that by $(2.4) \kappa \sim p$ ), is $\Delta t \lesssim \rho \Delta X^{2}\|D \boldsymbol{u}\| / p$. Since we use a time-implicit algorithm, these two latter upper bounds on the time step are only an indication of accuracy and are not needed for stability. Note that for an time-explicit algorithm, the last bound could be too restrictive (in particular if $\|D \boldsymbol{u}\|$ is small), and then should be replaced by the regularized one $\Delta t \lesssim \rho \epsilon \Delta X^{2} / p$, imposing $\Delta t$ to be proportional to $\epsilon$. This condition has been considered for stability reasons in the linearized implicit algorithm 
of [6]. With the above data, knowing that $\|\nabla \boldsymbol{u}\| \sim 1 \mathrm{~s}^{-1}, p \sim 10^{3} \mathrm{~Pa}$, the three above bounds lead to the values $1 \mathrm{~s}, 10^{-1} \mathrm{~s}, 10^{-4} s$ for $\Delta t$. This shows in passing that the plastic effects dominate over the viscous ones. These values motivate the choice $\Delta t_{\max }=\rho \Delta X^{2}\|D \boldsymbol{u}\| / p \approx$ $10^{-4} \mathrm{~s}$, except possibly if $\boldsymbol{D} \boldsymbol{u}$ is small, in which case we should take an even smaller time step. This difficulty is related to computing the interface position accurately, and this is why we use initially smaller time steps, as described in the last paragraph of Subsection 3.1.

Turning to the choice of $\epsilon$, it has to be taken sufficiently small so that the regularization error does not dominate over the space discretization error. According to [6, Theorem 4.7], in the inviscid case (the viscous effects are here negligible compared to plastic effects), the $\epsilon$-related error is proportional to $\sqrt{\epsilon T}$, where $T$ is the final time. This error has to be balanced with the second-order spatial error, which can be expected to be proportional to $\Delta X^{2}\|D \boldsymbol{u}\|^{2} \rho / p$. This leads to the choice $\epsilon \lesssim\left(\Delta X^{2}\|D \boldsymbol{u}\|^{2} \rho / p\right)^{2} / T$. This formula gives good results for the simple shear flow of Section 4. Taking here an average value $p / \rho \sim g \times 5 \mathrm{~cm}$ and $T=0.2 \mathrm{~s}$ leads to $\epsilon=2 \times 10^{-7} \mathrm{~s}^{-1}$. Our choice $\epsilon=10^{-6} \mathrm{~s}^{-1}$ is slightly greater than this value.

Initially the granular material is at rest and we take a vanishing (i.e. zero) initial velocity. The simulations and experiments proceed from $t=0 \mathrm{~s}$ to $t=1.02 \mathrm{~s}$, which corresponds to the time when the granular material has stopped in the experiments.

For the regularization method, the flowing part of the mass is defined to be the set of cells such that $|\boldsymbol{u}|>\varepsilon_{\text {stop }}$ with a velocity threshold $\varepsilon_{\text {stop }}=1 \mathrm{~mm} / \mathrm{s}$ which is approximately $10 \%$ of the typical variation of $\boldsymbol{u}$ over a cell (in the flowing region). The lower limit of this flowing part represents the static-flowing interface. Note that the value of $\varepsilon_{\text {stop }}$ is not related to the value of the regularization parameter $\epsilon$, but to the typical variation of $\boldsymbol{u}$ over a cell. The value of $\varepsilon_{\text {stop }}$ should not be taken too small owing to the numerical errors incurred in computing the velocity (in particular, discretization errors). We define the stopping time in the simulation as the time when all the cells are flagged to be in the static part. It turns out that the value of the stopping time is not sensitive to the value of $\varepsilon_{\text {stop }}$, since the stopping of the mass generally occurs swiftly. This is probably due to the fact that not only $D \boldsymbol{u}$ vanishes, but also $\boldsymbol{u}$ itself.

\subsection{Comparison with the augmented Lagrangian method}

Here we compare our results to those obtained with the augmented Lagrangian formulation described in [35]. We consider a trapezoidal and a rectangular geometry over a rigid bed, see Figure 6-(I)-(II). In this test, for both methods (regularization and augmented Lagrangian) we take a constant time step $\Delta t=10^{-3}$ s and a constant iteration stopping tolerance $\varepsilon_{\text {iter }}=10^{-3}$. We superimpose the profiles of the free surface obtained by each numerical method at different times. 

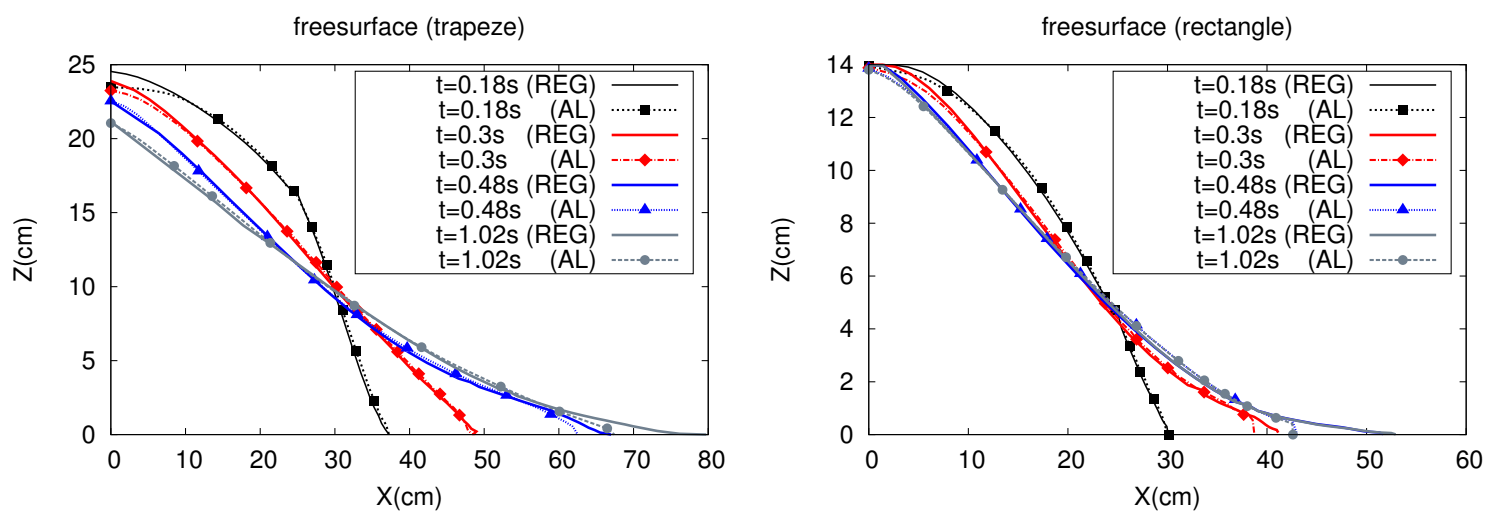

Figure 7: Evolution of the free surface calculated with the regularization method (full lines) and with the augmented Lagrangian method (dashed lines), at times $t=0.18 \mathrm{~s}, 0.3 \mathrm{~s}, 0.48 \mathrm{~s}$, 1.02s. Left: trapeze. Right: rectangle. The value $X=0$ corresponds to the left wall.

Figure 7 shows that the free surfaces calculated with the regularization and augmented Lagrangian methods are quite similar for both the trapezoidal and the rectangular geometries. We observe that the thickness of the mass at the left wall of the domain computed with the augmented Lagrangian method is lower than the one computed with the regularization method, and the regularization method leads to a slightly faster front propagation, i.e., a larger runout distance. In terms of computational time performance, the regularization method performs 7.8 times faster than the augmented Lagrangian method in the trapezoidal case, and 5 times faster in the rectangular case. 

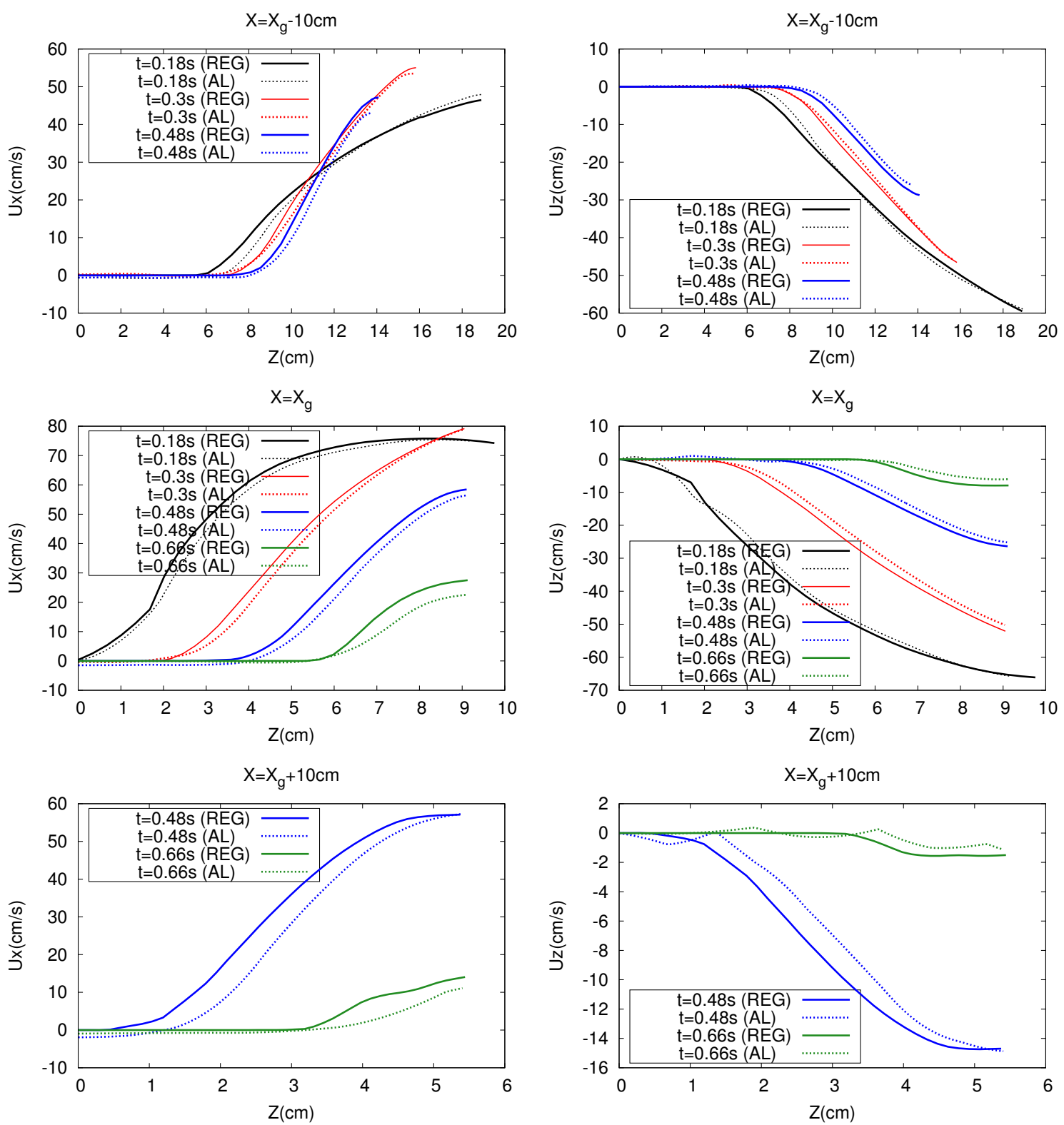

Figure 8: Comparison of the horizontal velocity profile $U_{X}$ (left) and vertical velocity profile $U_{Z}$ (right), for a trapezoidal geometry, at different times from $t=0.18 \mathrm{~s}$ to $t=0.66 \mathrm{~s}$, between the regularization method (full lines) and the augmented Lagrangian method (dotted lines), at three different vertical sections located at $X=X_{g}-10 \mathrm{~cm}, X=X_{g}$, and $X=X_{g}+10 \mathrm{~cm}$, where $X_{g}=29.7 \mathrm{~cm}$ is the position of the front of the released mass.

In the case of a trapezoidal geometry, we present the profiles of the two components of the velocity, $U_{X}$ and $U_{Z}$ as a function of $Z$, for three vertical sections located at $X=X_{g}-10 \mathrm{~cm}$, $X=X_{g}$, and $X=X_{g}+10 \mathrm{~cm}$, where $X_{g}=29.7 \mathrm{~cm}$ is the position of the front of the released mass. Figure 8 shows that both components of the velocity increase (in absolute value) with $Z$, and decrease with time except at the very beginning. The velocity profiles calculated by the two numerical methods are quite similar for each vertical section $X=X_{g}-10 \mathrm{~cm}$, $X=X_{g}$, and $X=X_{g}+10 \mathrm{~cm}$, and each time $(t=0.18 \mathrm{~s}$ to $t=0.66 \mathrm{~s})$. The absolute values of the horizontal and vertical velocities are however almost always slightly higher with the regularization method, especially at times $t=0.48 \mathrm{~s}$ and $t=0.66 \mathrm{~s}$, at $X=X_{g}+10 \mathrm{~cm}$. 

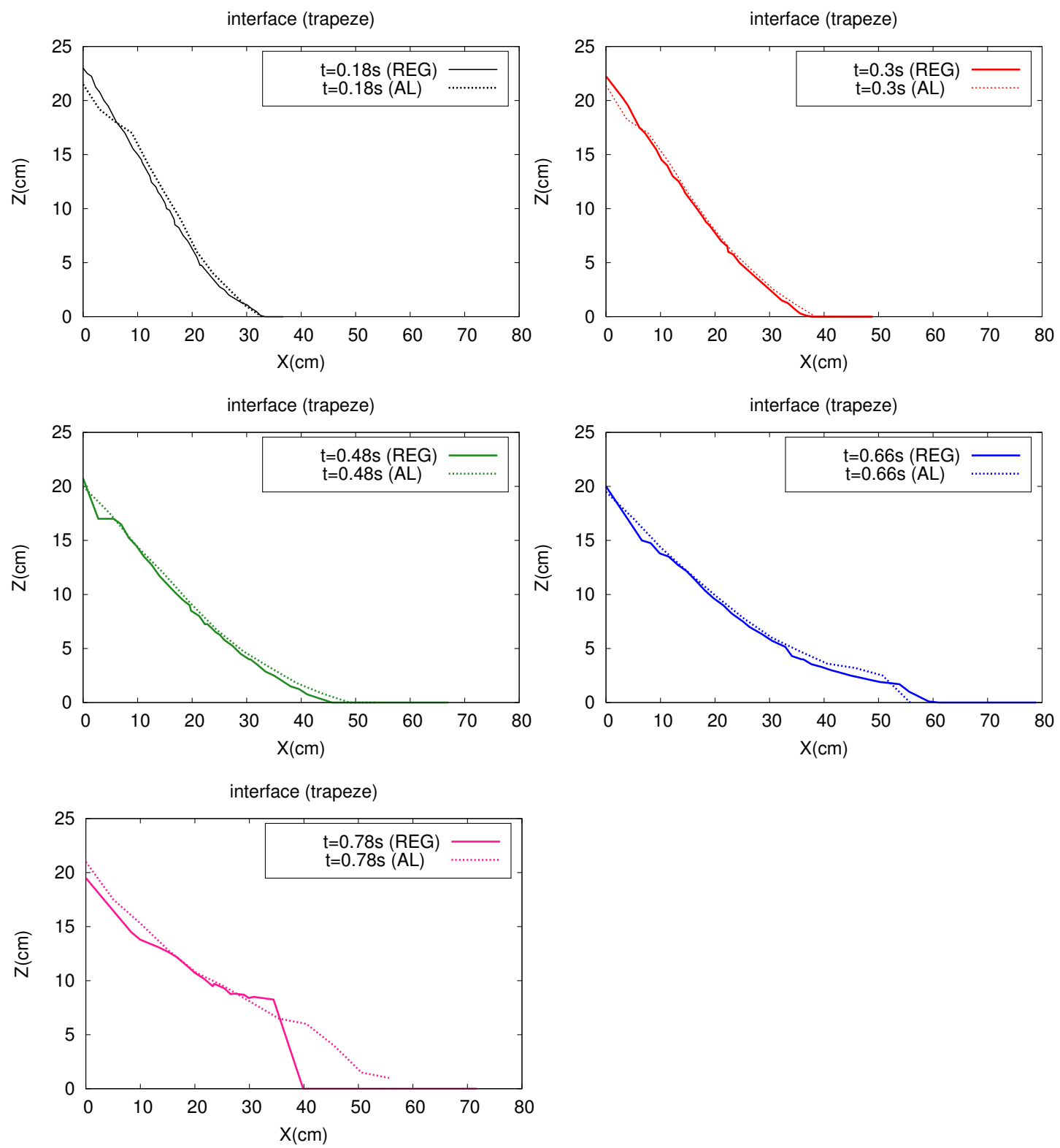

Figure 9: Comparison of the static-flowing interface $Z(t, X)$ at times $t=0.18 \mathrm{~s}, t=0.3 \mathrm{~s}, t=0.48 \mathrm{~s}$, $t=0.66 \mathrm{~s}, t=0.78 \mathrm{~s}$, between the regularization (full lines) and augmented Lagrangian methods (dotted lines).

Next, we compare the static-flowing interface obtained with the regularization and the augmented Lagrangian methods. Although the regularization method predicts a generally slightly lower position of the interface, both methods lead to similar results. The difference obtained at time $t=0.78 \mathrm{~s}$ can be explained by the fact that $\|D \boldsymbol{u}\|$ becomes very small at this time, and the domain is extremely narrow in the upstream part of the flow. Thus, it is difficult to extract the interface position with accuracy. This may explain why the front position calculated with the regularization method is slightly larger than that obtained with the augmented Lagrangian method used here (Figure 7).

To evaluate further the impact of the choice of $\epsilon$ in the regularization method, we run the simulation with the larger value $\epsilon=10^{-1} \mathrm{~s}^{-1}$. We plot the interface profiles on Figure 10. We observe that the larger value of $\epsilon$ leads to slightly rougher profiles. The difference is 
more noticeable on the left wall and at the front of the flowing mass. This effect of increasing $\epsilon$ is difficult to interpret. We can nevertheless observe that such an increase diminishes the magnitude of the stresses which have a stabilizing effect on the dynamics (for very large $\epsilon$, the governing equations get close to the incompressible Navier-Stokes equations for which instabilities typically arise in the present configuration). In any case, the position of the interface is still well captured, without any systematic deviation. The gain in computational time with respect to the previous test with $\epsilon=10^{-6} \mathrm{~s}^{-1}$ turns out to be only a factor of 1.2. We conclude that in this test case, the regularization method is not very sensitive to the choice of $\epsilon$ in the considered range, for accuracy as well as for computational time, except near the left wall and near the upstream part of the flow when the mass is close to stopping.
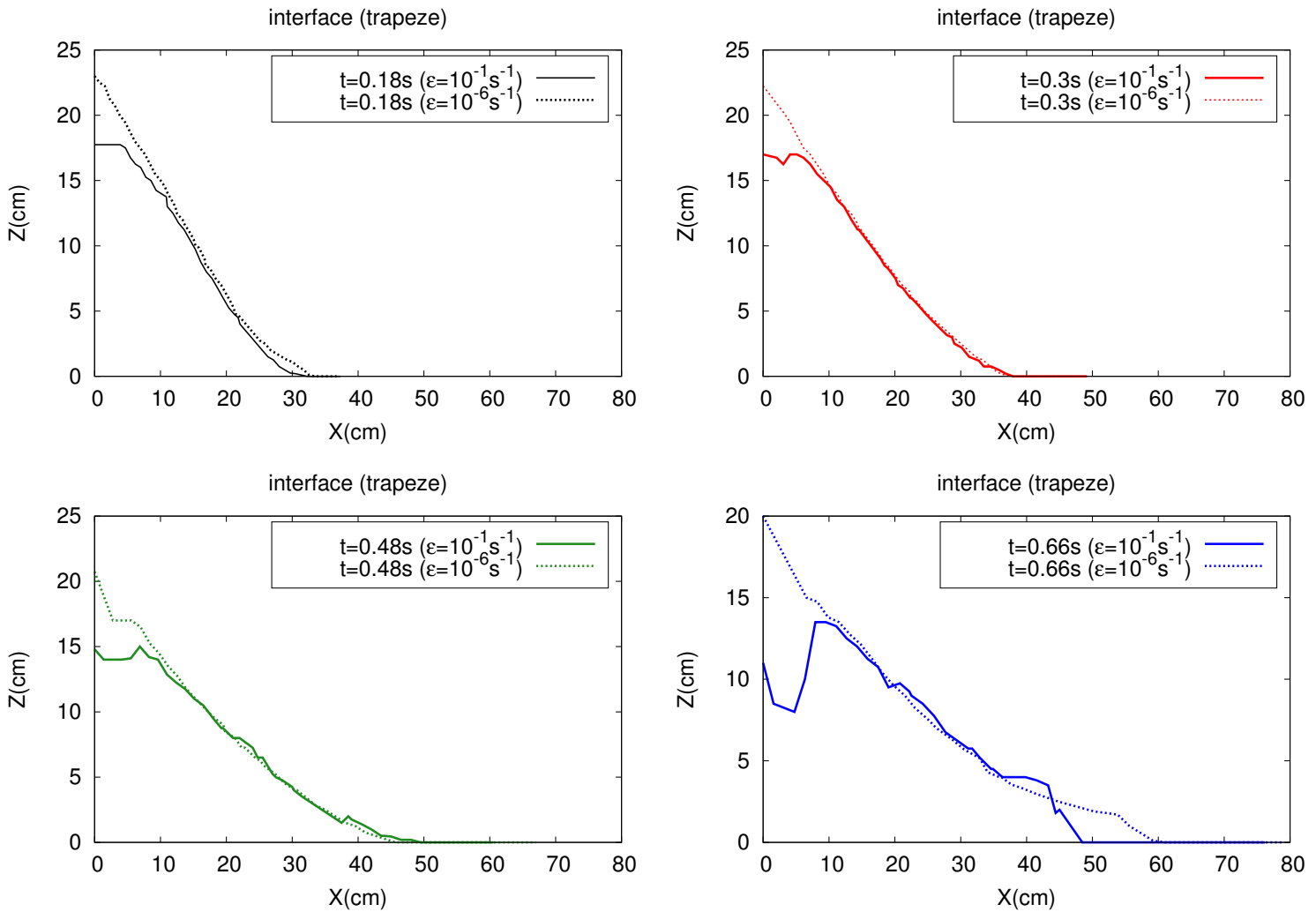

Figure 10: Comparison of the static-flowing interface $Z(t, X)$ at times $t=0.18 \mathrm{~s}, t=0.3 \mathrm{~s}, t=0.48 \mathrm{~s}$, $t=0.66 \mathrm{~s}$, between the regularization method with $\epsilon=10^{-1} \mathrm{~s}^{-1}$ and with $\epsilon=10^{-6} \mathrm{~s}^{-1}$.

\subsection{Comparison with laboratory experiments}

The experimental results presented here are processed from a series of experiments partially published in [25] where more details about the experiments and the methods of measurements made with a high-speed camera can be found. In particular, we have extracted here the evolution of the flow thickness, the velocity profiles, and the position of the static-flowing interface, in order to compare with the numerical simulations from the regularization method described in Section 3. The measurements of the velocity profile and of the static-flowing interface are not accurate, but provide an estimate of the time change of these quantities and of their spatial variations. In the line of the granular collapse experiments performed in $[51,25]$, we focus on two main configurations. The first one deals with a granular collapse over a rough rigid bed (Plexiglas covered by glued particles). The second one deals with a granular collapse over a thin erodible bed made of the same material and resting on a rough 
rigid substrate.

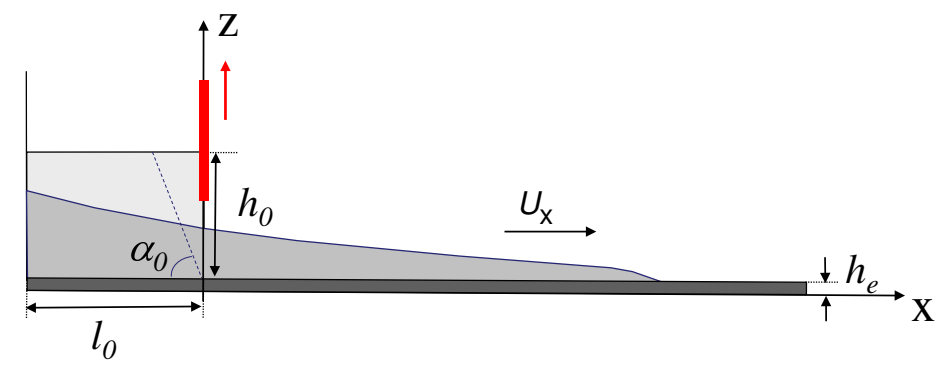

Figure 11: Experimental set-up: the initial mass with initial thickness $h_{0}=25 \mathrm{~cm}$, initial rectangle $\left(\alpha_{0}=90^{\circ}\right)$ or trapezoidal front angle $\alpha_{0}=70^{\circ}$, and initial width $l_{0}=30 \mathrm{~cm}$, is released and spreads (light gray) down a plane covered by an erodible bed of thickness $h_{e}=5 \mathrm{~mm}$, by opening very rapidly a gate (thick red line) at time $t=0$ s.

The experimental setup consists of a channel bordered by transparent plastic walls spaced by $10 \mathrm{~cm}$, topped with a reservoir. A granular mass in the reservoir is sustained by a sliding gate, see Figure 11. The reservoir shape is either trapezoidal (Figure 6-(I)), with a gate inclined at $70^{\circ}$ with respect to the horizontal, or rectangular (Figure 6-(II)) with a gate perpendicular to the channel base. Laboratory experiments made with erodible bed, (Figure 6-(III)), are performed on an erodible layer of $5 \mathrm{~mm}$ of thickness, made of the same material, initially set along the channel base. The granular mass is initially released from the reservoir by quickly opening the gate and spreads over the channel base.

\subsubsection{Collapse of a trapezoidal and a rectangular mass over a rigid bed}

We consider first the case 6-(I) of a trapezoidal granular mass over a rigid bed. We compare the evolution of the free surface (i.e., of the thickness profiles), of the velocity profiles $U_{X}, U_{Z}$ for two different vertical sections located at $X=X_{g}$ and $X=X_{g}+10 \mathrm{~cm}$ with $X_{g}=29.7 \mathrm{~cm}$, and of the position of the static-flowing interface. 

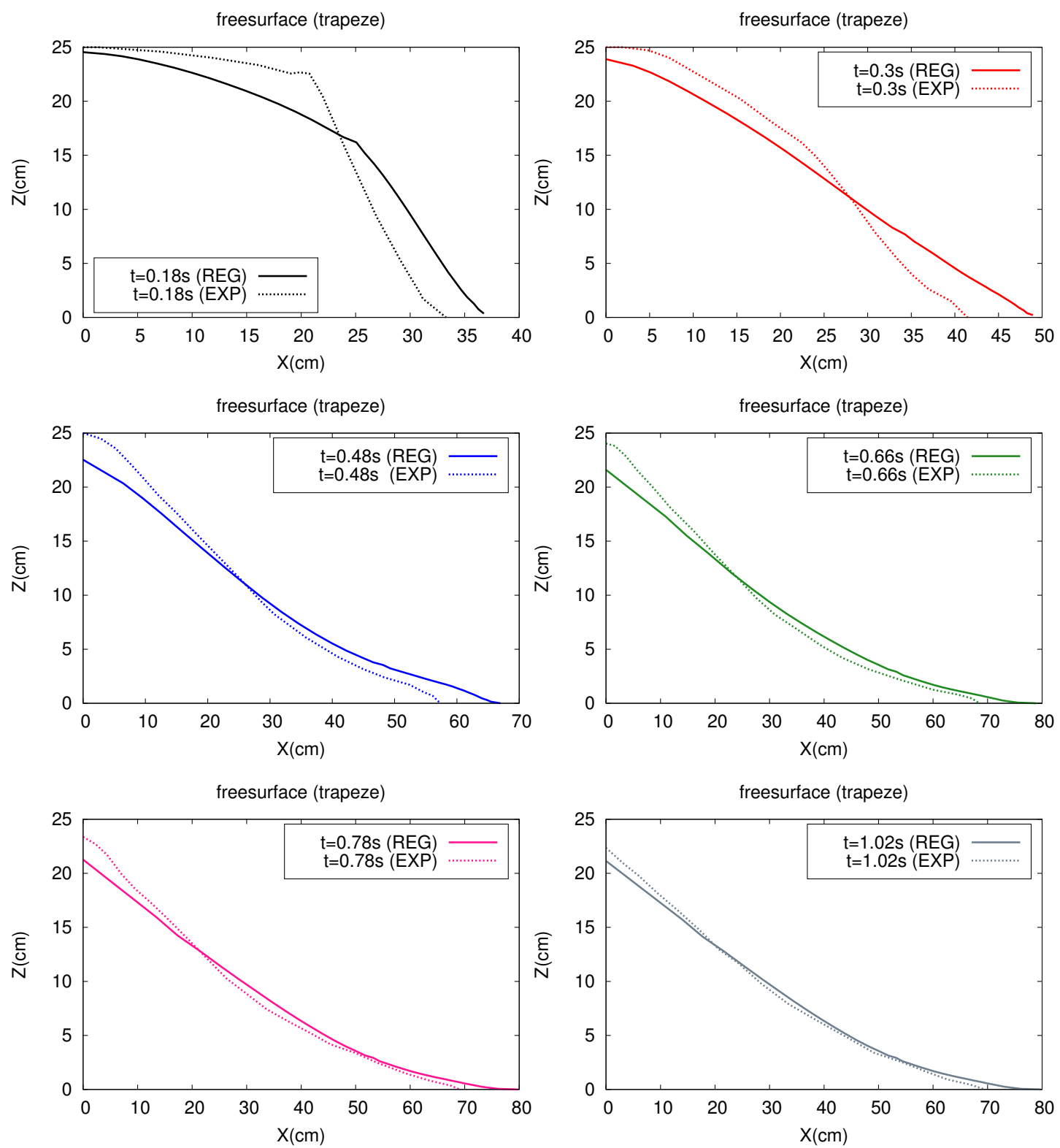

Figure 12: Comparison of the evolution of the free surface for a trapezoidal granular mass over a rigid bed (Figure 6-(I)), at times $t=0.18 \mathrm{~s}, t=0.3 \mathrm{~s}, t=0.48 \mathrm{~s}, t=0.66 \mathrm{~s}, t=0.78 \mathrm{~s}, t=1.02 \mathrm{~s}$, between the regularization method (full lines) and experimental results (dotted lines). The time at which the numerical mass stops is $t=0.86 \mathrm{~s}$.

The simulated thickness profiles (Figure 12) are in good agreement with the experimental observations, especially during the second part of the collapse $(t=0.66 \mathrm{~s}, t=0.78 \mathrm{~s}, t=1.02 \mathrm{~s})$, when the flow is close to stopping. The shape of the flow is very well reproduced, except near the left wall of the domain and near the front flow. On the left wall, the numerical thickness decreases more rapidly than the experimental one. However, it decreases less than the numerical thickness predicted by the augmented Lagrangian method. The position of the front is overestimated by the numerical simulations. These discrepancies are more visible during the starting phase of the flow. The final deposit is well reproduced, taking into account that the larger simulated runout involves a very thin thickness. Part of the discrepancy between the simulation and the experiments is due to the effect of removing the gate, as 
shown in [35] where the relative influence of the gate and of the rheology is discussed. Indeed, taking the gate into account slows down the mass spreading but leads to the same deposit.
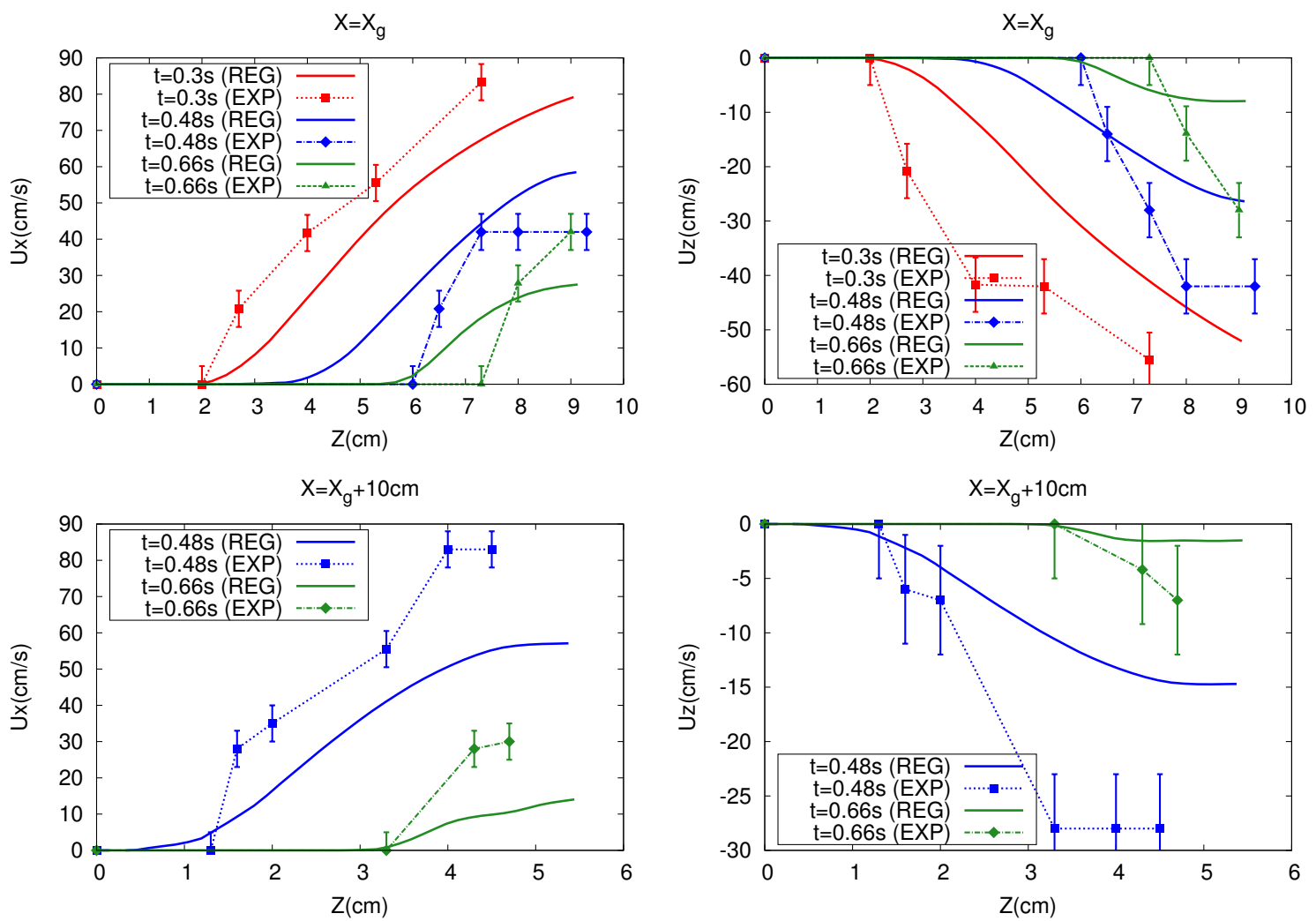

Figure 13: Comparison of the horizontal velocity profile $U_{X}$ (left) and of the vertical velocity profile $U_{Z}$ (right), for a trapezoidal geometry, at different times from $t=0.3 \mathrm{~s}$ to $t=0.66 \mathrm{~s}$, between the regularization method (full lines) and the experimental measurements (dotted lines plus error bars), for two different positions located at $X=X_{g}$ and $X=X_{g}+10 \mathrm{~cm}$, where the position of the gate in the experimental setup is $X_{g}=29.7 \mathrm{~cm}$.

To compare the velocity profiles $U_{X}$ and $U_{Z}$, we consider two vertical sections located at $X=X_{g}$ and $X=X_{g}+10 \mathrm{~cm}$. Figure 13 shows that the model qualitatively reproduces the change in time of velocity profiles, even though significant quantitative differences can be observed. The maximum horizontal velocity is reached close to the free surface. The inacurracy of the experimental measurements prevents however to perform a more precise comparison. 

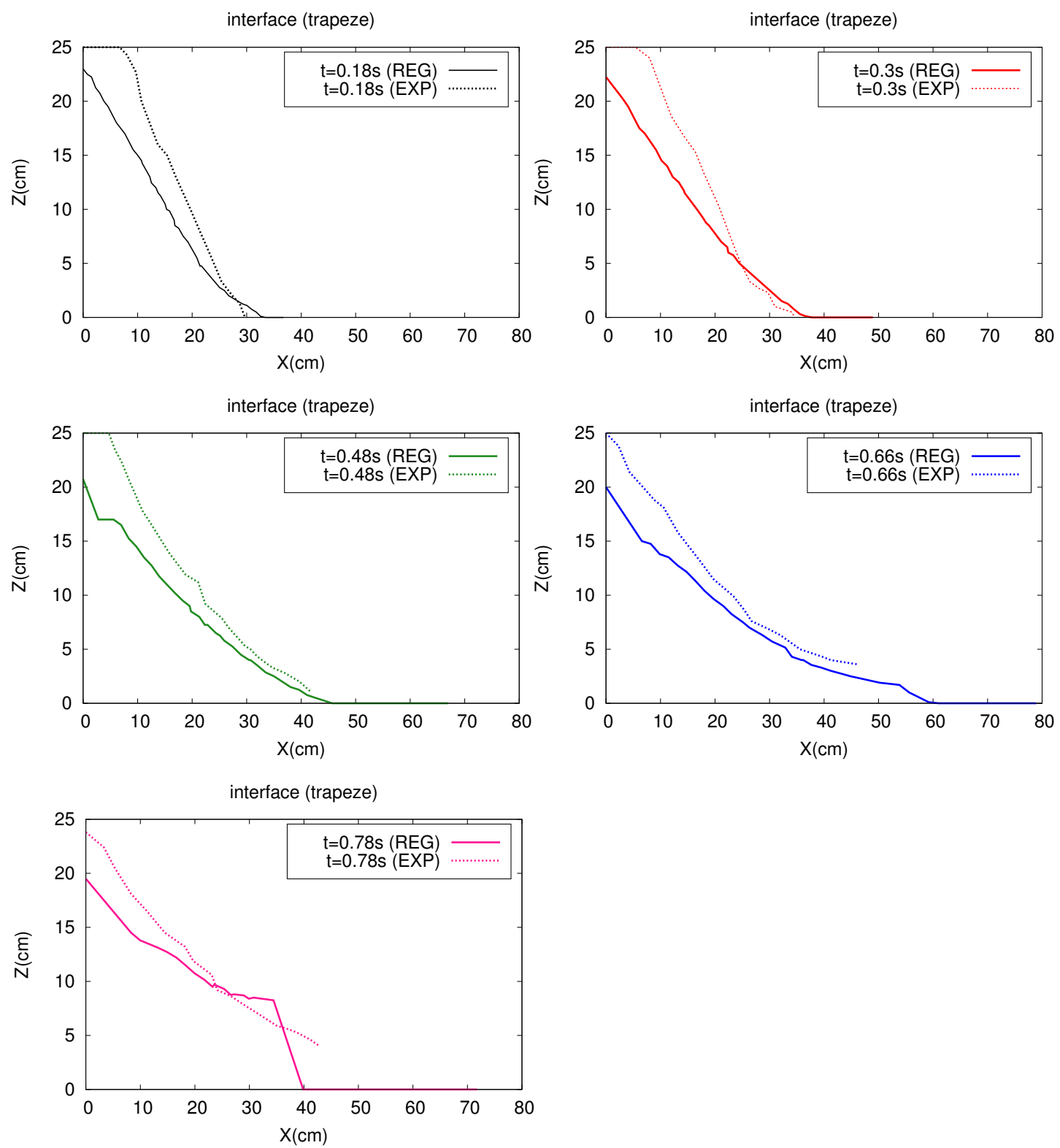

Figure 14: Comparison of the static-flowing interface $Z(t, X)$ at times $t=0.18 \mathrm{~s}, t=0.3 \mathrm{~s}, t=0.48 \mathrm{~s}$, $t=0.66 \mathrm{~s}, t=0.78 \mathrm{~s}$, between the regularization method (full lines) and the experimental measurements (dotted lines).

Next, we study the evolution of the static/flowing interface. Figure 14 shows that the position of the static-flowing interface within the granular mass is fairly well predicted numerically, except close to the left wall where the elevation of this interface is underestimated. The same qualitative difference was observed when comparing the regularization method with the augmented Lagrangian method (Figure 9). This underestimation of the position of the static-flowing interface may also be related to the fact that the gate is not taken into account here, leading to a faster flow dynamics than in the experiments. Furthermore the lateral wall friction has not been taken into account accurately here and may have a significant impact on the static/flowing interface position. Indeed, as the lateral wall friction increases with depth (with $p$ if Coulomb friction is assumed), this additional friction is expected to move the static/flowing interface upward. 

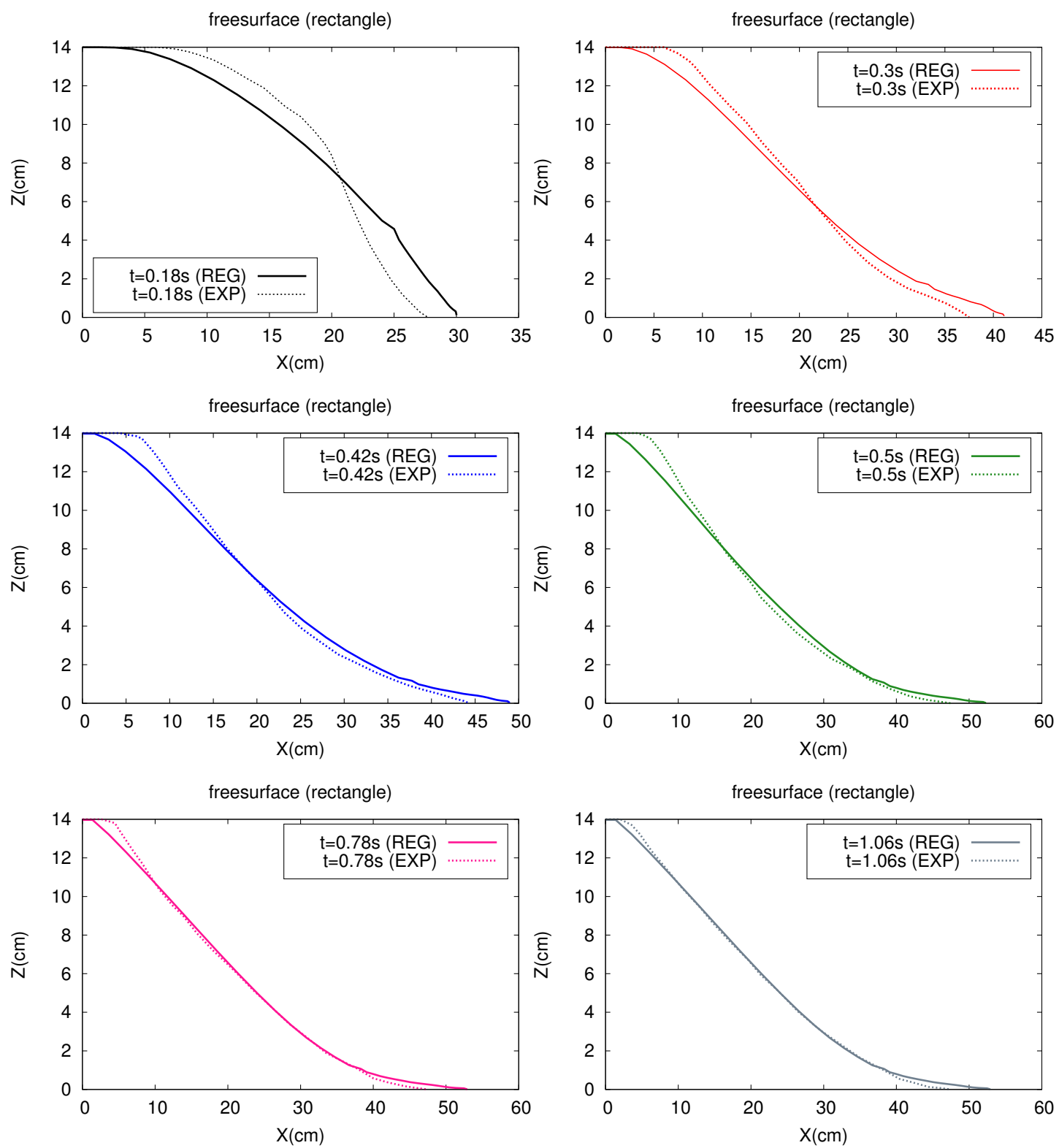

Figure 15: Comparison of the evolution of the free surface for a rectangular granular mass over a rigid bed (Figure 6-(II)), at times $t=0.18 \mathrm{~s}, t=0.3 \mathrm{~s}, t=0.42 \mathrm{~s}, t=0.5 \mathrm{~s}, t=0.78 \mathrm{~s}, t=$ $1.06 \mathrm{~s}$, between the regularization method (full lines) and the experimental measurements (dotted lines). The numerical mass stops around time $t=0.92 \mathrm{~s}$.

Similar results are obtained in the case of a rectangular granular mass released over a rigid bed (Figure 6-(II)), with an even better agreement between the simulated and experimental results. As shown on Figure 15, the collapse of a rectangular mass is quite well reproduced. Similarly to configuration 6-(I), the shape of the granular mass is very similar during its spreading over the rigid bed except on the left wall and close to the front of the flow. In the simulations, the thickness of the granular mass on the left wall is smaller, and the flow front propagates more rapidly than in the experiments. The shape of the final deposit and the runout distance are very well reproduced. 


\subsubsection{Collapse of a trapezoidal mass over an erodible bed}

In order to mimic real cases where erosion of material along the slope is often observed, we investigate the configuration of a granular collapse over an erodible bed made of the same material. The presence of an erodible layer along the spreading surface has been shown to change the flow dynamics and the deposit of the material over slopping beds, as investigated in $[13,21,25,37,50,51,52]$. For a granular collapse over an horizontal bed, the runout distance is only very slightly affected by the presence of the erodible bed.

Our purpose here is not to perform a precise analysis of the erosion processes, but to show that our numerical method is able to deal with the observed folding of the granular mass near the front. Indeed, without such method, simulation of granular collapses over erodible beds would be impossible. Thus, the present study can be viewed as a first step toward a more thorough numerical investigation of erosion processes over slopping beds. By comparing quantitatively our results to experiments [25], we show here that our method can reproduce the spreading of the granular mass over a thin, horizontal, erodible layer.

We consider the trapezoidal geometry, and the erodible bed is represented by a thin layer of thickness $h_{e}=5 \mathrm{~mm}$ under the trapezoidal column, see Figure 6-(III). Figure 16 shows that the numerical and experimental thickness profiles of the granular mass at different times are comparable all along the spreading of the mass. The final thickness profile and the shape of the deposit are also close. In contrast with the case of the rigid bed, at the beginning of the simulation, the front propagation is slower than in the experiments. Then, at times between $0.42 \mathrm{~s}$ and $0.5 \mathrm{~s}$, the numerical front catches up with the experimental front, whereas in the case of a rigid bed the front propagation is overestimated all along the simulation. Furthermore, the simulation does not reproduce the more rounded front obtained for flows over an erodible bed, compared to flows over a rigid bed. As a result, some processes may be missing in the rheology used here, such as a pressure and rate-dependent viscosity (as in the $\mu(I)$ rheology) that may be important for describing flows over an erodible bed. The numerical mass stops around $t=0.81 \mathrm{~s}$, earlier than in the experiments $(t=1.02 \mathrm{~s})$. Note that the mesh size is such that in the erodible bed there are vertically only two cells one above the other. A deeper study with more cells would probably be useful. 

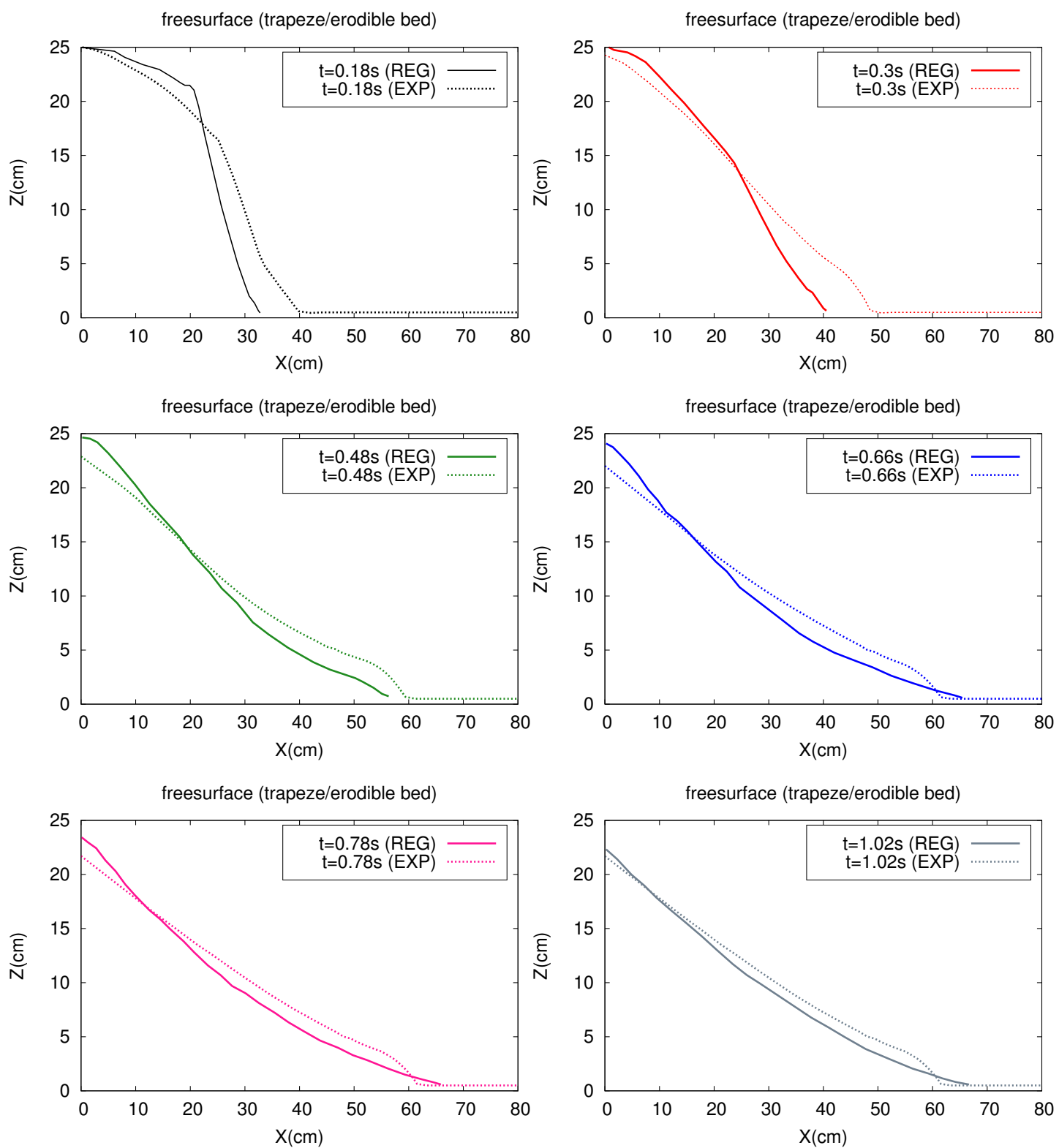

Figure 16: Comparison of the evolution of the free surface for a trapezoidal granular mass over an erodible bed of thickness $h_{e}=5 \mathrm{~mm}$, at times $t=0.18 \mathrm{~s}, t=0.3 \mathrm{~s}, t=0.48 \mathrm{~s}, t=0.66 \mathrm{~s}$, $t=0.78 \mathrm{~s}, t=1.02 \mathrm{~s}$, between regularization method (full lines) and experimental results (dotted lines).

\section{Conclusions}

We have proposed a regularization method together with an ALE method to deal with the displacement of the domain, for the simulation of two-dimensional flows of viscoplastic materials with pressure-dependent Drucker-Prager yield stress with free surface. In particular we have developed free surface folding treatments applicable for both rigid and erodible beds. We have set up a proper choice of the regularization parameter and the time step, that makes the regularization error negligible with respect to, or of the same order as the discretization error. We have verified our method on a simple shear flow configuration for which the solution can be computed by a 1D method, and on a granular collapse configuration by comparison 
to the results obtained by the augmented Lagrangian method. We have performed several comparisons with laboratory experiments of granular column collapse, showing for the first time that the regularization method combined with ALE-based free surface tracking is able to deal with such geophysically-relevant configurations with erodible bed. The regularization method has been shown to be more efficient in terms of computational time than the augmented Lagrangian method, while delivering results with similar accuracy with a suitable definition of the static/flowing interface.

\section{Acknowledgments}

The authors are thankful to Ioan Ionescu (University Paris 13) for stimulating discussions, especially on coding issues regarding ALE and the augmented Lagrangian method. This work has been partially funded by the French ANR program ANR-11-BS01-0016 LANDQUAKES, and by the ERC contract ERC-CG-2013-PE10-617472 SLIDEQUAKES.

\section{References}

[1] C. Ancey, Plasticity and geophysical flows: a review, J. Non-newtonian Fluid mech. 142 (2007), 4-35.

[2] B. Andreotti, Y. Forterre, O. Pouliquen, Les milieux granulaires, entre fluide et solide, Savoirs Actuels, EDP sciences, 2011.

[3] N. Balmforth, R. Kerswell, Granular collapse in two dimensions, J. Fluid Mech. 538 (2005), 399-428.

[4] T. Barker, D.G. Schaeffer, P. Bohorquez, J.M.N.T. Gray, Well-posed and ill-posed behaviour of the $\mu(I)$-rheology for granular flow, J. Fluid Mech. 779 (2015), 794-818.

[5] M. Bercovier, M. Engelman, A finite element method for incompressible non-newtonian flows, J. Comput. Phys. 36 (1980), 313-326.

[6] F. Bouchut, R. Eymard, A. Prignet, Convergence of conforming approximations for inviscid incompressible Bingham fluid flows and related problems, J. Evolution Eq. 14 (2014), 635-669.

[7] F. Bouchut, E. Fernandez-Nieto, A. Mangeney, P.-Y. Lagrée, On new erosion models of Savage-Hutter type for avalanches, Acta Mech. 199 (2008), 181-208.

[8] F. Bouchut, I.R. Ionescu, A. Mangeney, An analytic approach for the evolution of the static/flowing interface in viscoplastic granular flows, Comm. Math. Sci. 14 (2016), 21012126 .

[9] D. Bresch, E.D. Fernandez-Nieto, I. Ionescu, P. Vigneaux, Augmented Lagrangian method and compressible visco-plastic flows: applications to shallow dense avalanches, Advances in Mathematical Fluid Mechanics, New Directions in Mathematical Fluid Mechanics (2010), 57-89.

[10] C.J. Cawthorn, Several applications of a model for dense granular flows, PhD thesis, Univ. Cambdridge, 2011, http://www.repository.cam.ac.uk/handle/1810/245044.

[11] J. Chauchat, M. Médale, A three-dimensional numerical model for dense granular flows based on the $\mu(I)$ rheology, J. Comput. Phys. 256 (2014), 696-712.

[12] M. Costabel, A coercive bilinear form for Maxwell's equations, J. Math. Anal. Appl. 157 (1991), 527-541. 
[13] G.B. Crosta, S. Imposimato, D. Roddeman, Numerical modeling entrainment/deposition in rock and debris avalanches, Geol. 109 (2009), 135-145.

[14] G. B. Crosta, S. Imposimato, D. Roddeman, Numerical modeling of 2-D granular step collapse on erodible and nonerodible surface, J. Geophys. Res. 114 (2009), F03020.

[15] F. Da Cruz, S. Emam, M. Prochnow, J.-N. Roux, F. Chevoir, Rheophysics of dense granular materials: Discrete simulation of plane shear flows, Phys. Rev. E 72 (2005), 021309.

[16] P.G. De Gennes, Reflections on the mechanics of granular matter, Physica A 261 (1998), 267-293.

[17] R. Delannay, A. Valance, A. Mangeney, O. Roche, P. Richard, Granular and particleladen flows: from laboratory experiments to field observations, to appear in J. Phys. D: Appl. Phys., 2016.

[18] J.C. De los Reyes, S. González Andrade, Numerical simulation of two-dimensional Bingham fluid flow by semismooth Newton methods, J. Comput. Appl. Math. 235 (2010), 11-32.

[19] B. Domnik, S.P. Pudasaini, R. Katzenbach, S.A. Miller, Coupling of full two-dimensional and depth-averaged models for granular flows, J. Non-Newtonian Fluid Mech. 201 (2013), 56-68.

[20] D.C. Drucker, W. Prager, Soil mechanics and plastic analysis or limit design, Quarterly of Applied Mathematics 10 (1952), 157-165.

[21] A. Dufresne, Granular flow experiments on the interaction with stationary runout path materials and comparison to rock avalanche events, Earth Surf. Processes Landforms 37 (2012), 1527-1541.

[22] G. Duvaut, J.-L. Lions, Inequalities in mechanics and physic, Translated from the French by C. W. John, Grundlehren der Mathematischen Wissenschaften, 219, Springer-Verlag, Berlin, 1976.

[23] N. El Khouja, N. Roquet, B. Cazacliu, Analysis of a regularized Bingham model with pressure-dependent yield stress, J. Math. Fluid Mech. 17 (2015), 723-739.

[24] A. Ern, J.-L. Guermond, Theory and practice of finite elements, volume 159 of Applied Mathematical Sciences, Springer-Verlag, New York, NY, 2004.

[25] M. Farin, A. Mangeney, O. Roche, Fundamental changes of granular flow dynamics, deposition, and erosion processes at high slope angles: Insights from laboratory experiments, J. Geophys. Res. Earth Surf. 119 (2014), 504-532.

[26] P. Favreau, A. Mangeney, A. Lucas, G. Crosta, F. Bouchut, Numerical modeling of landquakes, Geophys. Res. Lett. 37 (2010), L15305.

[27] Y. Forterre, O. Pouliquen, Flows of dense granular media, Annu. Rev. Fluid Mech. 40 (2008), 1-24.

[28] M. Fortin, Calcul numérique des écoulements des fluides de Bingham et des fluides newtoniens incompressibles par la méthode des éléments finis, Thèse, Paris VI, 1972.

[29] I.A. Frigaard, C. Nouar, On the usage of viscosity regularisation methods for visco-plastic fluid flow computation, J. Non-Newtonian Fluid Mech. 127 (2005), 1-26.

[30] GDR MiDi group, On dense granular flows, Eur. Phys. J. E14 (2004), 341-365. 
[31] L. Girolami, V. Hergault, G. Vinay, A. Wachs, A three-dimensional discrete grain model for the simulation of dam-break rectangular collapses: comparison between numerical results and experiments, Granul. Matter 14 (2012), 381-392.

[32] R. Glowinski, P. Le Tallec, Augmented Lagrangian and operator-splitting methods in nonlinear mechanics, SIAM Stud. Appl. Math. 9, Philadelphia, PA, 1989.

[33] R. Glowinski, J.-L. Lions, R. Trémolières, Numerical analysis of variational inequalities, Studies in Mathematics and its Applications 8, North-Holland Publishing Co., Amsterdam, 1981.

[34] F. Hecht, New development in FreeFem++, J. Numer. Math. 20 (2012), 251-265. http://www.freefem.org/ff ++

[35] I.R. Ionescu, A. Mangeney, F. Bouchut, O. Roche, Viscoplastic modeling of granular column collapse with pressure-dependent rheology, J. Non-Newtonian Fluid Mech. 219 (2015), 1-18.

[36] I.R. Ionescu, Augmented Lagrangian for shallow viscoplastic flow with topography, J. Comput. Phys. 242 (2013), 544-560.

[37] R.M. Iverson, M. Reid, M. Logan, R. LaHusen, J.W. Godt, J. Griswold, Positive feedback and momentum growth during debris-flow entrainment of wet bed sediment, Nat. Geosci. 4 (2011), 116-121.

[38] R.M. Iverson, Elementary theory of bed-sediment entrainment by debris flows and avalanches, J. Geophys. Res. 117 (2012), F03006.

[39] R.M. Iverson, C. Ouyang, Entrainment of bed material by Earth-surface mass flows: review and reformulation of depth-integrated theory, Rev. Geophys. 53 (2015), 27-58.

[40] P. Jop, Y. Forterre, O. Pouliquen, Crucial role of sidewalls in dense granular flows: consequences for the rheology, J. Fluid Mech. 541 (2005), 167-192.

[41] P. Jop, Y. Forterre, O. Pouliquen, A constitutive law for dense granular flows, Nature 441 (2006), 727-730.

[42] P.-Y. Lagrée, L. Staron, S. Popinet, The granular column collapse as a continuum: validity of a two-dimensional Navier-Stokes model with a $\mu(I)$-rheology, J. Fluid Mech 686 (2011), 378-408.

[43] E. Lajeunesse, A. Mangeney-Castelnau, J.-P. Vilotte, Spreading of a granular mass on a horizontal plane, Phys. Fluids 16 (2004), 2371-2381.

[44] B.T. Liu, S.J. Muller, M.M. Denn, Convergence of a regularization method for creeping flow of a Bingham material about a rigid sphere, J. Non-Newtonian Fluid Mech. 102 (2002), 179-191.

[45] G. Lube, H.E. Huppert, R. Sparks, A. Freundt, Collapses of two-dimensional granular columns, Phys. Rev. E 72 (2005), 041301.

[46] A. Lucas, A. Mangeney, J.-P. Ampuero, Frictional weakening in landslides on earth and on other planetary bodies, Nature Communication 5 (2014), 3417.

[47] C. Lusso, Modélisation numérique des écoulements gravitaires viscoplastiques avec transition fluide/solide, $\mathrm{PhD}$ thesis. Université Paris-Est, Champs-sur-Marne, 2013. https://tel .archives-ouvertes.fr/tel-00977866v1

[48] C. Lusso, F. Bouchut, A. Ern, A. Mangeney, A free interface model for static/flowing dynamics in thin-layer flows of granular materials with yield: simple shear simulations and comparison to experiments, hal-00992309, 2016. 
[49] A. Mangeney-Castelnau, F. Bouchut, J.-P. Vilotte, E. Lajeunesse, A. Aubertin, M. Pirulli, On the use of Saint-Venant equations for simulating the spreading of a granular mass, J. Geophys. Res. 110 (2005), B09103.

[50] A. Mangeney, F. Bouchut, N. Thomas, J. P. Vilotte, M. O. Bristeau, Numerical modeling of self-channeling granular flows and of their levee-channel deposits, J. Geophys. Res. 112 (2007), F02017.

[51] A. Mangeney, O. Roche, O. Hungr, N. Mangold, G. Faccanoni, A. Lucas, Erosion and mobility in granular collapse over sloping beds, J. Geophys. Res. 115 (2010), F03040.

[52] A. Mangeney, L.S. Tsimring, D. Volfson, I.S Aranson, F. Bouchut, Avalanche mobility induced by the presence of an erodible bed and associated entrainment, Geophys. Res. Lett. 34 (2007), 22401.

[53] N. Martin, I.R. Ionescu, A. Mangeney, F. Bouchut, M. Farin, Continuum viscoplastic simulation of a granular column collapse on large slopes: $\mu(I)$ rheology and lateral wall effects, Phys. Fluids, 2017.

[54] L. Moretti, A. Mangeney, Y. Capdeville, E. Stutzmann, C. Christian Huggel, D. Schneider, F. Bouchut, Numerical modeling of the Mount Steller landslide flow history and of the generated long period seismic waves, Geophys. Res. Lett. 39 (2012), L16402.

[55] E. Ntovoris, M. Regis, A solution with free boundary for non-Newtonian fluids with Drucker-Prager plasticity criterion, hal-01374181, 2016.

[56] T.C. Papanastasiou, Flows of materials with yield, J. Rheology 31 (1987), 385-404.

[57] S. Pudasaini, K. Hutter, Avalanche Dynamics, Springer, Heidelberg, 2007.

[58] N. Roquet, P. Saramito, An adaptive finite element method for Bingham fluid flows around a cylinder, Comput. Methods Appl. Mech. Engrg. 192 (2003), 3317-3341.

[59] S.B. Savage, K. Hutter, The motion of a finite mass of granular material down a rough incline, Journal of fluid mechanics 199 (1989), 177-215.

[60] D.G. Schaeffer, Instability in the evolution equations describing incompressible granular flow, J. Differential Equations 66 (1987), 19-50.

[61] B. Sovilla, P. Burlando, P. Bartelt, Field experiments and numerical modeling of mass entrainment in snow avalanches, J. Geophys. Res. 111 (2006), F03007.

[62] R. Temam, Problèmes mathématiques en plasticité, Méthodes Mathématiques de l'Informatique 12, Gauthier-Villars, Montrouge, 1983.

[63] Y. Wang, Tracking of the yield surfaces of laminar viscoplastic flows in noncircular ducts using an adaptive scheme, Proceedings of the National Science Council, Republic of China, Part A: Physical Science and Engineering, vol. 23 (1999), 311-318. 